\title{
Semisimple Varieties of Implication Zroupoids
}

\author{
Juan M. CORNEJO *and Hanamantagouda P. SANKAPPANAVAR
}

\begin{abstract}
It is a well known fact that Boolean algebras can be defined using only implication and a constant. In 2012, this result was extended to De Morgan algebras in [8] which led Sankappanavar to introduce, and investigate, the variety $\mathbf{I}$ of implication zroupoids generalizing De Morgan algebras. His investigations were continued in [3] and [4] in which several new subvarieties of $\mathbf{I}$ were introduced and their relationships with each other and with the varieties of [8] were explored.

The present paper is a continuation of [8] and [3]. The main purpose of this paper is to determine the simple algebras in $\mathbf{I}$. It is shown that there are exactly five simple algebras in $\mathbf{I}$. From this description we deduce that the semisimple subvarieties of $\mathbf{I}$ are precisely the subvarieties of the variety generated by these 5 simple I-zroupoids and are locally finite. It also follows that the lattice of semisimple subvarieties of $\mathbf{I}$ is isomorphic to the direct product of a 4-element Boolean lattice and a 4-element chain.
\end{abstract}

\section{Introduction}

'It is a well known fact that Boolean algebras can be defined using only implication and a con'stant. In 2012, this result was extended to De Morgan algebras in [8] which led Sankappanavar to introduce, and investigate, the variety I of implication zroupoids generalizing De Morgan 'algebras. His investigations were continued in [3] and [4] in which several new subvarieties of I were introduced and their relationships with each other and with the varieties of [8] were explored.

The present paper is a continuation of $[8$ and [3]. The main purpose of this paper is to 'determine the simple algebras in $\mathbf{I}$. It is shown that there are five simple algebras in $\mathbf{I}$, namely:

- the 2-element trivial implication zroupoid $\mathbf{2}_{\mathbf{z}}$, where $x \rightarrow y:=0$

- the 2-element $\vee$-semilattice $\mathbf{2}_{\mathbf{s}}$ with the least element 0 ,

- the 2-element Boolean algebra $\mathbf{2}_{\mathbf{b}}$,

- the 3 -element Kleene algebra $\mathbf{3}_{\mathbf{k}}$, and

- the 4-element De Morgan algebra $\mathbf{4}_{\mathrm{d}}$.

From this description it follows that the semisimple subvarieties of $\mathbf{I}$ are precisely the subvarieties of the variety $\mathbb{V}\left(\mathbf{2}_{\mathrm{z}}, \mathbf{2}_{\mathrm{s}}, \mathbf{2}_{\mathrm{b}}, \mathbf{3}_{\mathrm{k}}, \mathbf{4}_{\mathrm{d}}\right)$ generated by the 5 simple I-zroupoids and are locally finite. It also follows that the lattice of semisimple varieties of implication zroupoids is isomorphic to the direct product of a 4-element Boolean lattice and a 4-element chain.

\footnotetext{
${ }^{*}$ I wish to dedicate this work to my little daughter Catalina Cornejo
} 
The method employed in this paper to describe simple algebras of $\mathbf{I}$ will, we hope, inspire a way for solving the problem of characterizing all the subdirectly irreducible members of $\mathbf{I}$ and, hence, for obtaining information about the lattice of subvarieties of the variety of implication zroupoids.

We include an appendix with some of the longer proofs so that the reader can focus on the main ideas and results of the paper.

\section{Preliminaries}

For the concepts not defined, and results not proved, in this paper we refer the reader to the textbooks [1, [2], and [7]. The familiarity with [3] and [8], although not necessary, is helpful to the reader. We recall from [8] the following definition which is central to this paper.

Definition 2.1 An algebra $\mathbf{A}=\langle A, \rightarrow, 0\rangle$, where $\rightarrow$ is binary and 0 is a constant, is called a zroupoid. A zroupoid $\mathbf{A}=\langle A, \rightarrow, 0\rangle$ is an Implication zroupoid (I-zroupoid, for short) if $\mathbf{A}$ satisfies:

(I) $(x \rightarrow y) \rightarrow z \approx\left[\left(z^{\prime} \rightarrow x\right) \rightarrow(y \rightarrow z)^{\prime}\right]^{\prime}$, where $x^{\prime}:=x \rightarrow 0$

$\left(\mathrm{I}_{0}\right) \quad 0^{\prime \prime} \approx 0$.

Throughout this paper $\mathbf{I}$ denotes the variety of implication zroupoids.

In this paper we use the characterizations of De Morgan algebras, Kleene algebras and Boolean algebras obtained in [8] as definitions.

Definition 2.2 An implication zroupoid $\mathbf{A}=\langle A, \rightarrow, 0\rangle$ is a De Morgan algebra (DM-algebra for short) if $\mathbf{A}$ satisfies the axiom:

$(\mathrm{DM})(x \rightarrow y) \rightarrow x \approx x$.

A DM-algebra $\mathbf{A}=\langle A, \rightarrow, 0\rangle$ is a Kleene algebra (KL-algebra for short) if $\mathbf{A}$ satisfies the axiom:

$\left(\mathrm{KL}_{1}\right)(x \rightarrow x) \rightarrow(y \rightarrow y)^{\prime} \approx x \rightarrow x$

or, equivalently,

$\left(\mathrm{KL}_{2}\right)(y \rightarrow y) \rightarrow(x \rightarrow x) \approx x \rightarrow x$.

A DM-algebra $\mathbf{A}=\langle A, \rightarrow, 0\rangle$ is a Boolean algebra (BA-algebra for short) if $\mathbf{A}$ satisfies the axiom:

(BA) $x \rightarrow x \approx 0^{\prime}$.

We denote by $\mathbf{D M}, \mathbf{K L}, \mathbf{B A}$ respectively the varieties of $\mathbf{D M}$-algebras, $\mathbf{K L}$-algebras and $\mathbf{B A}$ algebras. 


\section{Some properties of Implication Zroupoids in $\mathbf{I}_{2,0}$}

Recall from [8] that $\mathbf{I}_{\mathbf{2}, \mathbf{0}}$ denotes the subvariety of $\mathbf{I}$ defined by the identity: $x^{\prime \prime} \approx x$. In this section, in addition to recalling some known results, we present some new properties of $\mathbf{I}_{\mathbf{2}, \mathbf{0}}$ which, being of interest in their own right, will be useful later.

Lemma $\mathbf{3 . 1}$ [8] Let $\mathbf{A} \in \mathbf{I}_{\mathbf{2}, \mathbf{0}}$. Then

(a) $x^{\prime} \rightarrow 0^{\prime} \approx 0 \rightarrow x$

(b) $0 \rightarrow x^{\prime} \approx x \rightarrow 0^{\prime}$

Lemma 3.2 [8, Theorem 8.15] Let $\mathbf{A}$ be an $\mathbf{I}$-zroupoid. Then the following are equivalent:

(a) $0^{\prime} \rightarrow x \approx x$

(b) $x^{\prime \prime} \approx x$

(c) $\left(x \rightarrow x^{\prime}\right)^{\prime} \approx x$

(d) $x^{\prime} \rightarrow x \approx x$.

The following lemma, whose proof is given in the Appendix, plays a crucial role in the rest of the paper.

Lemma 3.3 Let $\mathbf{A} \in \mathbf{I}_{\mathbf{2}, \mathbf{0}}$. Then:

(1) $\left(x \rightarrow 0^{\prime}\right) \rightarrow y \approx\left(x \rightarrow y^{\prime}\right) \rightarrow y$

(2) $\left(0 \rightarrow x^{\prime}\right) \rightarrow(y \rightarrow x) \approx y \rightarrow x$

(3) $x \rightarrow(0 \rightarrow x)^{\prime} \approx x^{\prime}$

(4) $(y \rightarrow x)^{\prime} \approx(0 \rightarrow x) \rightarrow(y \rightarrow x)^{\prime}$

(5) $\left[x \rightarrow(y \rightarrow x)^{\prime}\right]^{\prime} \approx(x \rightarrow y) \rightarrow x$

(6) $(y \rightarrow x) \rightarrow y \approx(0 \rightarrow x) \rightarrow y$

(7) $0 \rightarrow x \approx 0 \rightarrow(0 \rightarrow x)$

(8) $0 \rightarrow\left(x \rightarrow y^{\prime}\right)^{\prime} \approx 0 \rightarrow\left(x^{\prime} \rightarrow y\right)$

(9) $[(x \rightarrow y) \rightarrow x] \rightarrow[(y \rightarrow x) \rightarrow y] \approx x \rightarrow y$

(10) $\left[x^{\prime} \rightarrow(0 \rightarrow y)\right]^{\prime} \approx(0 \rightarrow x) \rightarrow(0 \rightarrow y)^{\prime}$

(11) $0 \rightarrow(0 \rightarrow x)^{\prime} \approx 0 \rightarrow x^{\prime}$

(12) $0 \rightarrow\left(x^{\prime} \rightarrow y\right)^{\prime} \approx x \rightarrow\left(0 \rightarrow y^{\prime}\right)$

(13) $0 \rightarrow(x \rightarrow y) \approx x \rightarrow(0 \rightarrow y)$

(14) $[(0 \rightarrow x) \rightarrow y] \rightarrow x \approx\left[(y \rightarrow x) \rightarrow(0 \rightarrow x)^{\prime}\right]^{\prime}$

(15) $(x \rightarrow y) \rightarrow(0 \rightarrow y)^{\prime} \approx(x \rightarrow y)^{\prime}$ 
(16) $x \rightarrow\left[\left(y \rightarrow z^{\prime}\right) \rightarrow x\right]^{\prime} \approx(0 \rightarrow y) \rightarrow\left\{(0 \rightarrow z) \rightarrow x^{\prime}\right\}$

(17) $(x \rightarrow y) \rightarrow y^{\prime} \approx y \rightarrow(x \rightarrow y)^{\prime}$

(18) $0 \rightarrow\left\{(0 \rightarrow x) \rightarrow y^{\prime}\right\} \approx x \rightarrow\left(0 \rightarrow y^{\prime}\right)$

(19) $\left[\left(x \rightarrow 0^{\prime}\right) \rightarrow y\right]^{\prime} \approx(0 \rightarrow x) \rightarrow y^{\prime}$

(20) $[(0 \rightarrow x) \rightarrow y] \rightarrow x \approx y \rightarrow x$

(21) $(0 \rightarrow x) \rightarrow(0 \rightarrow y) \approx x \rightarrow(0 \rightarrow y)$

(22) $0 \rightarrow\left[(x \rightarrow y) \rightarrow z^{\prime}\right]^{\prime} \approx(x \rightarrow y)^{\prime} \rightarrow(0 \rightarrow z)$

$(23)\left[\left(x^{\prime} \rightarrow y^{\prime}\right) \rightarrow(y \rightarrow x)^{\prime}\right] \rightarrow(y \rightarrow x) \approx y \rightarrow x$

(24) $\left[0 \rightarrow\left(x^{\prime} \rightarrow y^{\prime}\right)^{\prime}\right] \rightarrow(y \rightarrow x) \approx y \rightarrow x$

(25) $[0 \rightarrow(x \rightarrow y)] \rightarrow x \approx(0 \rightarrow y) \rightarrow x$

(26) $(0 \rightarrow x) \rightarrow(x \rightarrow y) \approx x \rightarrow(x \rightarrow y)$

(27) $(0 \rightarrow x) \rightarrow[y \rightarrow(0 \rightarrow x)]^{\prime} \approx[y \rightarrow(0 \rightarrow x)]^{\prime}$

(28) $[(x \rightarrow y) \rightarrow(0 \rightarrow x)]^{\prime} \approx(0 \rightarrow x) \rightarrow\left[y \rightarrow(0 \rightarrow x)^{\prime}\right]$

(29) $(x \rightarrow y) \rightarrow(0 \rightarrow x) \approx y \rightarrow(0 \rightarrow x)$

(30) $y \rightarrow(y \rightarrow x) \approx[x \rightarrow(0 \rightarrow y)] \rightarrow(y \rightarrow x)$

(31) $x \rightarrow y \approx x \rightarrow(x \rightarrow y)$

$(32)[\{x \rightarrow(0 \rightarrow y)\} \rightarrow z]^{\prime} \approx z \rightarrow[(x \rightarrow y) \rightarrow z]^{\prime}$

(33) $\left[0 \rightarrow(x \rightarrow y)^{\prime}\right] \rightarrow y \approx(0 \rightarrow x) \rightarrow y$

(34) $\left[\left\{x \rightarrow\left(0 \rightarrow(y \rightarrow z)^{\prime}\right)\right\} \rightarrow z\right]^{\prime} \approx\left(z^{\prime} \rightarrow x\right) \rightarrow\left[z \rightarrow(y \rightarrow z)^{\prime}\right]$

(35) $x \rightarrow\left[\left\{y \rightarrow(z \rightarrow x)^{\prime}\right\} \rightarrow x\right]^{\prime} \approx\left(x^{\prime} \rightarrow y\right) \rightarrow\left[x \rightarrow(z \rightarrow x)^{\prime}\right]$

(36) $[0 \rightarrow(x \rightarrow y)] \rightarrow y^{\prime} \approx y \rightarrow(x \rightarrow y)^{\prime}$

(37) $\left(x^{\prime} \rightarrow y\right) \rightarrow\left[x \rightarrow(z \rightarrow x)^{\prime}\right] \approx(0 \rightarrow y) \rightarrow\left[x \rightarrow(z \rightarrow x)^{\prime}\right]$

(38) $\left[x^{\prime} \rightarrow(y \rightarrow z)\right] \rightarrow\left[x \rightarrow(y \rightarrow x)^{\prime}\right] \approx x \rightarrow[(z \rightarrow y) \rightarrow x]^{\prime}$

(39) $x \rightarrow[(y \rightarrow z) \rightarrow x]^{\prime} \approx[0 \rightarrow(z \rightarrow y)] \rightarrow\left[x \rightarrow(z \rightarrow x)^{\prime}\right]$

$(40)[0 \rightarrow(x \rightarrow y)] \rightarrow\left[z \rightarrow(x \rightarrow z)^{\prime}\right] \approx(0 \rightarrow y) \rightarrow\left[z \rightarrow(x \rightarrow z)^{\prime}\right]$

(41) $x \rightarrow[(y \rightarrow z) \rightarrow x]^{\prime} \approx(0 \rightarrow y) \rightarrow\left[x \rightarrow(z \rightarrow x)^{\prime}\right]$

(42) $0 \rightarrow[(0 \rightarrow x) \rightarrow y] \approx x \rightarrow(0 \rightarrow y)$

(43) $x \rightarrow(y \rightarrow x)^{\prime} \approx\left(y \rightarrow 0^{\prime}\right) \rightarrow x^{\prime}$

(44) if $\left(x \rightarrow y^{\prime}\right) \rightarrow x=x$ then $\left(x^{\prime} \rightarrow y\right) \rightarrow x^{\prime}=x^{\prime}$. 
$(45)\left[\left(0 \rightarrow x^{\prime}\right) \rightarrow y\right] \rightarrow\left[0 \rightarrow(z \rightarrow x)^{\prime}\right] \approx\left[\{y \rightarrow(0 \rightarrow z)\} \rightarrow\left(x \rightarrow 0^{\prime}\right)^{\prime}\right]^{\prime}$

(46) $[\{x \rightarrow(0 \rightarrow y)\} \rightarrow z]^{\prime} \approx(0 \rightarrow x) \rightarrow\left[z \rightarrow(y \rightarrow z)^{\prime}\right]$

(47) $\left[\left(0 \rightarrow x^{\prime}\right) \rightarrow y\right] \rightarrow\left[0 \rightarrow(z \rightarrow x)^{\prime}\right] \approx y \rightarrow\left(0 \rightarrow(z \rightarrow x)^{\prime}\right)$

(48) $[(0 \rightarrow x) \rightarrow y] \rightarrow(0 \rightarrow z) \approx 0 \rightarrow[(x \rightarrow y) \rightarrow z]$

(49) $\left(x^{\prime} \rightarrow y\right) \rightarrow\left[0 \rightarrow(z \rightarrow x)^{\prime}\right] \approx y \rightarrow\left[0 \rightarrow(z \rightarrow x)^{\prime}\right]$

(50) $y \rightarrow\left[0 \rightarrow(z \rightarrow x)^{\prime}\right] \approx 0 \rightarrow\left[\left(x^{\prime} \rightarrow y\right) \rightarrow(z \rightarrow x)^{\prime}\right]$

(51) $(0 \rightarrow x) \rightarrow\left[\{0 \rightarrow(y \rightarrow z)\} \rightarrow u^{\prime}\right] \approx[0 \rightarrow\{(x \rightarrow y) \rightarrow z\}] \rightarrow u^{\prime}$

(52) $[0 \rightarrow\{(x \rightarrow y) \rightarrow z\}]^{\prime} \approx(0 \rightarrow x) \rightarrow\left[\left(0 \rightarrow y^{\prime}\right) \rightarrow(0 \rightarrow z)^{\prime}\right]$

(53) $0 \rightarrow\left[(x \rightarrow y) \rightarrow\left(z \rightarrow x^{\prime}\right)^{\prime}\right] \approx 0 \rightarrow\left[y \rightarrow\left(z^{\prime} \rightarrow x\right)\right]$

(54) $0 \rightarrow\left[(x \rightarrow y)^{\prime} \rightarrow z\right] \approx 0 \rightarrow\left[x \rightarrow\left(y^{\prime} \rightarrow z\right)\right]$

$(55)\left[\{(x \rightarrow y) \rightarrow z\} \rightarrow\{0 \rightarrow(y \rightarrow z)\}^{\prime}\right] \rightarrow[(x \rightarrow y) \rightarrow z] \approx(x \rightarrow y) \rightarrow z$

(56) $\left[0 \rightarrow(x \rightarrow y)^{\prime}\right] \rightarrow(z \rightarrow y) \approx(0 \rightarrow x) \rightarrow(z \rightarrow y)$

(57) $\left[x \rightarrow\left(y^{\prime} \rightarrow z\right)^{\prime}\right] \rightarrow x \approx\left[y \rightarrow\left\{0 \rightarrow(0 \rightarrow z)^{\prime}\right\}\right] \rightarrow x$

(58) $[(0 \rightarrow x) \rightarrow y] \rightarrow(z \rightarrow x) \approx y \rightarrow(z \rightarrow x)$

(59) $x \rightarrow[(y \rightarrow x) \rightarrow y] \approx x \rightarrow y$

(60) $[(x \rightarrow y) \rightarrow(y \rightarrow z)]^{\prime} \approx(0 \rightarrow x) \rightarrow(y \rightarrow z)^{\prime}$

(61) $(x \rightarrow y) \rightarrow(y \rightarrow x) \approx y \rightarrow x$

(62) $\left[\{(x \rightarrow y) \rightarrow z\} \rightarrow\left(z^{\prime} \rightarrow x\right)^{\prime}\right] \rightarrow[(x \rightarrow y) \rightarrow z] \approx(x \rightarrow y) \rightarrow z$

(63) $\left[\{(x \rightarrow y) \rightarrow z\} \rightarrow\left\{z^{\prime} \rightarrow(y \rightarrow x)\right\}^{\prime}\right] \rightarrow[(x \rightarrow y) \rightarrow z] \approx(x \rightarrow y) \rightarrow z$.

\section{Simple algebras in the variety $I_{2,0}$}

In this section we will prove that if $\mathbf{A} \in \mathbf{I}_{\mathbf{2}, \mathbf{0}}$ is a simple algebra with $|A| \geq 3$, then $\mathbf{A} \in \mathbf{D M}$.

Definition 4.1 Let $\mathbf{A} \in \mathbf{I}_{\mathbf{2}, \mathbf{0}}$. We define the relation $R_{1}$ on $\mathbf{A}$ as follows:

$$
x R_{1} y \text { if and only if }\left(x \rightarrow y^{\prime}\right) \rightarrow x=x \text { and }\left(y \rightarrow x^{\prime}\right) \rightarrow y=y .
$$

Lemma 4.2 Let $\mathbf{A} \in \mathbf{I}_{\mathbf{2}, \mathbf{0}}$. Then $R_{1}$ is a equivalence relation on $\mathbf{A}$.

Proof Let $a \in A$. By Lemma 3.2 (d) we have that $\left(a \rightarrow a^{\prime}\right) \rightarrow a=\left(a^{\prime \prime} \rightarrow a^{\prime}\right) \rightarrow a=a^{\prime} \rightarrow a=a$. Then the relation $R_{1}$ is reflexive. The symmetry is immediate. Now we show that $R_{1}$ is transitive. Let $a, b, c \in A$ and assume that $a R_{1} b$ and $b R_{1} c$. This implies that

(4.1) $\left(a \rightarrow b^{\prime}\right) \rightarrow a=a$, 
$(4.2)\left(b \rightarrow a^{\prime}\right) \rightarrow b=b$,

$(4.3)\left(b \rightarrow c^{\prime}\right) \rightarrow b=b$

and

(4.4) $\left(c \rightarrow b^{\prime}\right) \rightarrow c=c$.

By Lemma 3.3 (44) and Lemma (4.1) we have

(4.5) $a^{\prime}=\left(a^{\prime} \rightarrow b\right) \rightarrow a^{\prime}$.

Therefore,

$$
\begin{aligned}
& a^{\prime}=\left(a^{\prime} \rightarrow b\right) \rightarrow a^{\prime} \\
& =(0 \rightarrow b) \rightarrow a^{\prime} \\
& =\left(0 \rightarrow\left(\left(b \rightarrow c^{\prime}\right) \rightarrow b\right)\right) \rightarrow a^{\prime} \\
& =\left[0 \rightarrow\left\{\left(0 \rightarrow c^{\prime}\right) \rightarrow b\right\}\right] \rightarrow a^{\prime} \\
& =\left[\left(0 \rightarrow c^{\prime}\right) \rightarrow(0 \rightarrow b)\right] \rightarrow a^{\prime} \\
& =\left[\left\{a^{\prime \prime} \rightarrow\left(0 \rightarrow c^{\prime}\right)\right\} \rightarrow\left\{(0 \rightarrow b) \rightarrow a^{\prime}\right\}^{\prime}\right]^{\prime} \text { by }(\mathrm{I}) \\
& =\left[\left\{a \rightarrow\left(0 \rightarrow c^{\prime}\right\}\right] \rightarrow\left\{(0 \rightarrow b) \rightarrow a^{\prime}\right\}^{\prime}\right]^{\prime} \\
& =\left[\left\{a \rightarrow\left(0 \rightarrow c^{\prime}\right)\right\} \rightarrow\left\{\left(a^{\prime} \rightarrow b\right) \rightarrow a^{\prime}\right\}^{\prime}\right]^{\prime} \text { by Lemma 3.3 (6) } \\
& =\left[\left\{a \rightarrow\left(0 \rightarrow c^{\prime}\right)\right\} \rightarrow a^{\prime \prime}\right]^{\prime} \quad \text { by (4.5) } \\
& =\left[\left\{a \rightarrow\left(0 \rightarrow c^{\prime}\right)\right\} \rightarrow a\right]^{\prime} \\
& =\left[\left\{0 \rightarrow\left(0 \rightarrow c^{\prime}\right)\right\} \rightarrow a\right]^{\prime} \quad \text { by Lemma } 3.3 \text { (16) } \\
& =\left[\left(0 \rightarrow c^{\prime}\right) \rightarrow a\right]^{\prime} \\
& =\left[\left(a \rightarrow c^{\prime}\right) \rightarrow a\right]^{\prime}
\end{aligned}
$$

and, hence we get

$$
a=a^{\prime \prime}=\left[\left(a \rightarrow c^{\prime}\right) \rightarrow a\right]^{\prime \prime}=\left(a \rightarrow c^{\prime}\right) \rightarrow a .
$$

Similary, from (4.2) and (4.4), we conclude that

$$
\left(c \rightarrow a^{\prime}\right) \rightarrow c=c,
$$

implying $a R_{1} c$.

Lemma 4.3 Let $\mathbf{A} \in \mathbf{I}_{\mathbf{2}, \mathbf{0}}$. Then $R_{1}$ is a congruence.

Proof By Lemma 4.2, $R_{1}$ is an equivalence relation. Let $a, b, c, d \in A$ satisfying $a R_{1} b$ and $c R_{1} d$. Then

(4.6) $\left(a \rightarrow b^{\prime}\right) \rightarrow a=a$,

(4.7) $\left(b \rightarrow a^{\prime}\right) \rightarrow b=b$,

(4.8) $\left(c \rightarrow d^{\prime}\right) \rightarrow c=c$

and

(4.9) $\left(d \rightarrow c^{\prime}\right) \rightarrow d=d$. 
From (4.6) and (4.8), and Lemma 3.3 (44), we have that

(4.10) $\left(a^{\prime} \rightarrow b\right) \rightarrow a^{\prime}=a^{\prime}$.

and

(4.11) $\left(c^{\prime} \rightarrow d\right) \rightarrow c^{\prime}=c^{\prime}$.

Consequently,

$$
\begin{aligned}
a^{\prime} \rightarrow\left[\left(b \rightarrow a^{\prime}\right)^{\prime}\right] & =\left[a^{\prime} \rightarrow\left[\left(b \rightarrow a^{\prime}\right)^{\prime}\right]\right]^{\prime \prime} & & \\
& =\left[\left(a^{\prime} \rightarrow b\right) \rightarrow a^{\prime}\right]^{\prime} & & \text { by Lemma 3.3 (5) } \\
& =a^{\prime \prime} & & \text { using (4.10) } \\
& =a . & &
\end{aligned}
$$

Therefore,

(4.12) $a^{\prime} \rightarrow\left[\left(b \rightarrow a^{\prime}\right)^{\prime}\right]=a$.

Hence we have

$$
\begin{aligned}
(0 \rightarrow b) \rightarrow(a \rightarrow c) & =\left(b^{\prime} \rightarrow 0^{\prime}\right) \rightarrow(a \rightarrow c) \\
& =\left[(b \rightarrow 0) \rightarrow 0^{\prime}\right] \rightarrow(a \rightarrow c) \\
& =\left[\left(b \rightarrow 0^{\prime \prime}\right) \rightarrow 0^{\prime}\right] \rightarrow(a \rightarrow c) \\
& =\left[\left(b \rightarrow 0^{\prime}\right) \rightarrow 0^{\prime}\right] \rightarrow(a \rightarrow c) \\
& =\left[\left(b \rightarrow 0^{\prime}\right) \rightarrow(a \rightarrow c)^{\prime}\right] \rightarrow(a \rightarrow c) \\
& =\left[\left\{b \rightarrow(a \rightarrow c)^{\prime \prime}\right\} \rightarrow(a \rightarrow c)^{\prime}\right] \rightarrow(a \rightarrow c) \\
& =\left[\{b \rightarrow(a \rightarrow c)\} \rightarrow(a \rightarrow c)^{\prime}\right] \rightarrow(a \rightarrow c) \\
& =\left[\{b \rightarrow(a \rightarrow c)\} \rightarrow 0^{\prime}\right] \rightarrow(a \rightarrow c) \\
& =[\{b \rightarrow(a \rightarrow c)\} \rightarrow(0 \rightarrow 0)] \rightarrow(a \rightarrow c) \\
& =[\{0 \rightarrow(b \rightarrow(a \rightarrow c)\}](0 \rightarrow 0)] \rightarrow(a \rightarrow c) \\
& =[0 \rightarrow[[0 \rightarrow(b \rightarrow(a \rightarrow c))] \rightarrow 0]] \rightarrow(a \rightarrow c) \\
& =[(a \rightarrow c) \rightarrow[[0 \rightarrow(b \rightarrow(a \rightarrow c))] \rightarrow 0]] \rightarrow(a \rightarrow c) \\
& =\left[(a \rightarrow c) \rightarrow[0 \rightarrow(b \rightarrow(a \rightarrow c))]^{\prime}\right] \rightarrow(a \rightarrow c) \\
& =\left[(a \rightarrow c) \rightarrow\left[0 \rightarrow\left[\left(b \rightarrow a^{\prime}\right)^{\prime} \rightarrow c\right]\right]^{\prime}\right] \rightarrow(a \rightarrow c)
\end{aligned}
$$

by Lemma 3.1 (b)

by Lemma 3.3 (11) by Lemma 3.3 (1) by Lemma 3.3 (1)

by Lemma 3.3 (1)

by Lemma 3.3 (21) by Lemma 3.3 (13) by Lemma 3.3 (6) by Lemma 3.3 (154) with $x=b, y=a^{\prime}, z=c$

Then

$(4.13)(0 \rightarrow b) \rightarrow(a \rightarrow c)=\left[(a \rightarrow c) \rightarrow\left[0 \rightarrow\left[\left(b \rightarrow a^{\prime}\right)^{\prime} \rightarrow c\right]\right]^{\prime}\right] \rightarrow(a \rightarrow c)$.

By (4.13) and (4.12), we have

$(0 \rightarrow b) \rightarrow(a \rightarrow c)=\left[\left(\left[a^{\prime} \rightarrow\left[\left(b \rightarrow a^{\prime}\right)^{\prime}\right]\right] \rightarrow c\right) \rightarrow\left[0 \rightarrow\left[\left(b \rightarrow a^{\prime}\right)^{\prime} \rightarrow c\right]\right]^{\prime}\right] \rightarrow\left(\left[a^{\prime} \rightarrow\left[\left(b \rightarrow a^{\prime}\right)^{\prime}\right]\right] \rightarrow c\right)$.

From (4.14) and Lemma 3.3 (55) with $x=a^{\prime}, y=\left(b \rightarrow a^{\prime}\right)^{\prime}$ and $z=c$ we conclude that

$$
(0 \rightarrow b) \rightarrow(a \rightarrow c)=\left[a^{\prime} \rightarrow\left[\left(b \rightarrow a^{\prime}\right)^{\prime}\right]\right] \rightarrow c .
$$

Therefore, by (4.12), we have

(4.15) $(0 \rightarrow b) \rightarrow(a \rightarrow c)=a \rightarrow c$. 
Consequently, in view of Lemma 3.3 (56) , we can conclude that $a \rightarrow c=\left[0 \rightarrow(b \rightarrow c)^{\prime}\right] \rightarrow(a \rightarrow$ c). From Lemma 3.3 (6), we obtain

$$
a \rightarrow c=\left[(a \rightarrow c) \rightarrow(b \rightarrow c)^{\prime}\right] \rightarrow(a \rightarrow c) .
$$

Similarly, in view of (4.7), we have

$$
b \rightarrow c=\left[(b \rightarrow c) \rightarrow(a \rightarrow c)^{\prime}\right] \rightarrow(b \rightarrow c) .
$$

Thus we conclude

(4.16) $a \rightarrow c R_{1} b \rightarrow c$.

Moreover, from Lemma 3.3 (2), we have

$$
b \rightarrow c=\left(0 \rightarrow c^{\prime}\right) \rightarrow(b \rightarrow c)
$$

Now, we observe that

$$
\begin{aligned}
b \rightarrow c & =\left(0 \rightarrow c^{\prime}\right) \rightarrow(b \rightarrow c) \\
& =\left[\left(0 \rightarrow c^{\prime}\right) \rightarrow(b \rightarrow c)\right]^{\prime} \rightarrow\left[\left(0 \rightarrow c^{\prime}\right) \rightarrow(b \rightarrow c)\right] \\
& =\left[\left(0 \rightarrow\left(\left(c^{\prime} \rightarrow d\right) \rightarrow c^{\prime}\right)\right) \rightarrow(b \rightarrow c)\right]^{\prime} \rightarrow\left[\left(0 \rightarrow c^{\prime}\right) \rightarrow(b \rightarrow c)\right] \\
& =\left[\left(0 \rightarrow\left((0 \rightarrow d) \rightarrow c^{\prime}\right)\right) \rightarrow(b \rightarrow c)\right]^{\prime} \rightarrow\left[\left(0 \rightarrow c^{\prime}\right) \rightarrow(b \rightarrow c)\right] \\
& =\left[\left((0 \rightarrow d) \rightarrow\left(0 \rightarrow c^{\prime}\right)\right) \rightarrow(b \rightarrow c)\right]^{\prime} \rightarrow\left[\left(0 \rightarrow c^{\prime}\right) \rightarrow(b \rightarrow c)\right] \\
& =\left[\left(d \rightarrow\left(0 \rightarrow c^{\prime}\right)\right) \rightarrow(b \rightarrow c)\right]^{\prime} \rightarrow\left[\left(0 \rightarrow c^{\prime}\right) \rightarrow(b \rightarrow c)\right] \\
& =\left[\left[(b \rightarrow c)^{\prime} \rightarrow d\right] \rightarrow\left[\left(0 \rightarrow c^{\prime}\right) \rightarrow(b \rightarrow c)\right]^{\prime}\right]^{\prime \prime} \rightarrow\left[\left(0 \rightarrow c^{\prime}\right) \rightarrow(b \rightarrow c)\right] \\
& =\left[\left[(b \rightarrow c)^{\prime} \rightarrow d\right] \rightarrow\left[\left(0 \rightarrow c^{\prime}\right) \rightarrow(b \rightarrow c)\right]^{\prime} \rightarrow\left[\left(0 \rightarrow c^{\prime}\right) \rightarrow(b \rightarrow c)\right]\right. \\
& =\left[\left[(b \rightarrow c)^{\prime} \rightarrow d\right] \rightarrow 0^{\prime}\right] \rightarrow\left[\left(0 \rightarrow c^{\prime}\right) \rightarrow(b \rightarrow c)\right] \\
& =\left[0 \rightarrow\left[(b \rightarrow c)^{\prime} \rightarrow d\right]^{\prime}\right] \rightarrow\left[\left(0 \rightarrow c^{\prime}\right) \rightarrow(b \rightarrow c)\right] \\
& =\left[\left[\left(0 \rightarrow c^{\prime}\right) \rightarrow(b \rightarrow c)\right] \rightarrow\left[(b \rightarrow c)^{\prime} \rightarrow d\right]^{\prime}\right] \rightarrow\left[\left(0 \rightarrow c^{\prime}\right) \rightarrow(b \rightarrow c)\right] \\
& =\left[[b \rightarrow c] \rightarrow\left[(b \rightarrow c)^{\prime} \rightarrow d\right]^{\prime}\right] \rightarrow[b \rightarrow c] \\
& =\left[[b \rightarrow c] \rightarrow\left[0 \rightarrow(0 \rightarrow d)^{\prime}\right]\right] \rightarrow[b \rightarrow c] \\
& =\left[0 \rightarrow\left[0 \rightarrow(0 \rightarrow d)^{\prime}\right]\right] \rightarrow[b \rightarrow c] \\
& =\left[0 \rightarrow(0 \rightarrow d)^{\prime}\right] \rightarrow[b \rightarrow c] \\
& =\left(0 \rightarrow d^{\prime}\right) \rightarrow[b \rightarrow c] \\
& =\left[(b \rightarrow c)^{\prime \prime} \rightarrow\left[d^{\prime} \rightarrow(b \rightarrow c)\right]^{\prime}\right]^{\prime} \\
& =\left[(b \rightarrow c) \rightarrow\left[d^{\prime} \rightarrow(b \rightarrow c)\right]^{\prime}\right]^{\prime} \\
& =\left[(0 \rightarrow d) \rightarrow\left[(b \rightarrow c) \rightarrow(0 \rightarrow(b \rightarrow c))^{\prime}\right]\right]^{\prime} \\
& =\left[(0 \rightarrow d) \rightarrow(b \rightarrow c)^{\prime}\right]^{\prime} \\
& =[(d \rightarrow b) \rightarrow(b \rightarrow c)]^{\prime \prime} \\
= & (d \rightarrow b) \rightarrow(b \rightarrow c)
\end{aligned}
$$

by (4.17)

by Lemma 3.2 (d) by (4.11)

by Lemma 3.3 (6) by Lemma 3.3 (13) by Lemma 3.3 (21) by (I)

by Lemma 3.3 (1) by Lemma 3.1 (b) by Lemma 3.3 (6) by (4.17) by Lemma 3.3 (57) with $x=b \rightarrow c, y=b \rightarrow c, z=d$ by Lemma 3.3 (6) by Lemma 3.3 (31) by Lemma 3.3 (11) by (I)

by Lemma 3.3 (41) with $x=b \rightarrow c, y=d, z=0$ by Lemma 3.3 (159) with $x=b \rightarrow c, y=0$ by Lemma 3.3 (60)

Consequently,

$$
b \rightarrow c=(d \rightarrow b) \rightarrow(b \rightarrow c)
$$


Hence,

$$
\begin{aligned}
b \rightarrow c & =(d \rightarrow b) \rightarrow(b \rightarrow c) \\
& =\left[[(d \rightarrow b) \rightarrow(b \rightarrow c)] \rightarrow\left[(b \rightarrow c)^{\prime} \rightarrow(b \rightarrow d)\right]^{\prime}\right] \rightarrow[(d \rightarrow b) \rightarrow(b \rightarrow c)] \\
& =\left[[b \rightarrow c] \rightarrow\left[(b \rightarrow c)^{\prime} \rightarrow(b \rightarrow d)\right]^{\prime}\right] \rightarrow[b \rightarrow c] \\
& =\left[0 \rightarrow\left[(b \rightarrow c)^{\prime} \rightarrow(b \rightarrow d)\right]^{\prime}\right] \rightarrow(b \rightarrow c) \\
& =\left[0 \rightarrow\left[\left[(b \rightarrow d)^{\prime} \rightarrow(b \rightarrow c)\right] \rightarrow[0 \rightarrow(b \rightarrow d)]^{\prime}\right]^{\prime \prime}\right] \rightarrow(b \rightarrow c) \\
& =\left[0 \rightarrow\left[\left[(b \rightarrow d)^{\prime} \rightarrow(b \rightarrow c)\right] \rightarrow[0 \rightarrow(b \rightarrow d)]^{\prime}\right]\right] \\
& =\left[(b \rightarrow c) \rightarrow\left[0 \rightarrow(0 \rightarrow(b \rightarrow d))^{\prime}\right]\right] \rightarrow(b \rightarrow c) \\
& =\left[(b \rightarrow c) \rightarrow\left[0 \rightarrow(b \rightarrow d)^{\prime}\right]\right] \rightarrow(b \rightarrow c) \\
& =\left[0 \rightarrow\left[0 \rightarrow(b \rightarrow d)^{\prime}\right]\right] \rightarrow(b \rightarrow c) \\
& =\left(0 \rightarrow(b \rightarrow d)^{\prime}\right) \rightarrow(b \rightarrow c) \\
& =\left((b \rightarrow c) \rightarrow(b \rightarrow d)^{\prime}\right) \rightarrow(b \rightarrow c)
\end{aligned}
$$

by (4.18)

by Lemma $3.3(63)$ with $x=d, y=b, z=b \rightarrow c$ by (4.18) by Lemma 3.3 (6) by (I)

by Lemma 3.3 (150) with $x=b \rightarrow d, y=b \rightarrow c, z=0$ by Lemma 3.3 (11) by Lemma 3.3 (6) by Lemma 3.3 (31) by Lemma 3.3 (6)

In a similar way, by (4.9), we have that

$$
b \rightarrow d=\left((b \rightarrow d) \rightarrow(b \rightarrow c)^{\prime}\right) \rightarrow(b \rightarrow d) .
$$

Then

(4.19) $b \rightarrow c R_{1} b \rightarrow d$.

In view of (4.16), (4.19) and Lemma (4.2), we conclude $a \rightarrow c R_{1} b \rightarrow d$.

Lemma 4.4 Let $\mathbf{A} \in \mathbf{I}_{2,0}$ be a simple algebra with $|A| \geq 3$, and let $a, b \in A$ with $a, b \neq 0$. Then

(a) If $0=0^{\prime}$, then $0 \rightarrow a \neq 0, a \rightarrow 0 \neq 0, a \rightarrow b \neq 0$.

(b) $0^{\prime} \neq 0$.

\section{Proof}

(a) Let $a, b \in A$ with $a, b \neq 0$. Assume that $0 \rightarrow a=0$. Then $0=0 \rightarrow a=0^{\prime} \rightarrow a=a$ by Lemma 3.2 (国), which is a contradiction, implying $0 \rightarrow a \neq 0$.

If $a \rightarrow 0=0$ then $a=a^{\prime \prime}=(a \rightarrow 0)^{\prime}=0^{\prime}=0$, a contradiction.

If $a \rightarrow b=0$, then, by Lemma 3.3 (31), we have $0=a \rightarrow b=a \rightarrow(a \rightarrow b)=a \rightarrow 0$, a contradiction.

(b) Assume that $0=0^{\prime}$. Considerer the equivalence relation, $R_{2}$ associated to the partition $\left\{\{0\}, A^{*}\right\}$ of $A$ with $A^{*}=\{c \in A: c \neq 0\}$. Since $|A| \geq 3$, we observe that $R_{2} \neq \Delta, A \times A$. Now, let $a, b, c, d \in A$ satisfying $a R_{2} b$ and $c R_{2} d$. From $0^{\prime}=0$ and item (国) we conclude that $R_{2}$ is a congruence, which is a contradiction, because $\mathbf{A}$ is simple. Thus $0^{\prime} \neq 0$.

We are now ready to present our main theorem of this section.

Theorem 4.5 Let $\mathbf{A} \in \mathbf{I}_{\mathbf{2}, \mathbf{0}}$ be a simple algebra with $|A| \geq 3$. Then $\mathbf{A} \in \mathbf{D M}$. 
Proof We know, in view of Lemma 4.3, that the relation $R_{1}$, defined in Definition 4.1, is a congruence on $\mathbf{A}$. We also know that $0^{\prime} \neq 0$ from Lemma 4.4 (b). Observe that

$$
\left(0 \rightarrow 0^{\prime \prime}\right) \rightarrow 0=(0 \rightarrow 0) \rightarrow 0=0^{\prime \prime}=0
$$

and

$$
\left(0^{\prime} \rightarrow 0^{\prime}\right) \rightarrow 0^{\prime}=0^{\prime} \rightarrow 0^{\prime}=0,^{\prime}
$$

by Lemma 3.2 (国). Hence $0 R_{1} 0^{\prime}$. Since $\mathbf{A}$ is simple and $0^{\prime} \neq 0$, it follows that $R_{1}=A \times A$. Therefore,

$$
A \models\left(x \rightarrow y^{\prime}\right) \rightarrow x \approx x .
$$

Thus, letting $a, b \in A$, we can conclude that $(a \rightarrow b) \rightarrow a=\left(a \rightarrow b^{\prime \prime}\right) \rightarrow a=a$. Consequently, we have proved

$$
A \models\left(x \rightarrow y^{\prime}\right) \rightarrow x \approx x,
$$

implying that $\mathbf{A} \in \mathbf{D M}$.

\section{Semisimple Implication Zroupoids}

In this section we dermine the semisimple subvarieties of $\mathbf{I}$.

Recall from [8] that there are three 2-element algebras in $\mathbf{I}$, namely $\mathbf{2}_{\mathbf{z}}, \mathbf{2}_{\mathbf{s}}, \mathbf{2}_{\mathbf{b}}$, whose $\rightarrow$ operations are, respectively, as follows:

\begin{tabular}{r|rr}
$\rightarrow:$ & 0 & 1 \\
\hline 0 & 0 & 0 \\
1 & 0 & 0
\end{tabular}

\begin{tabular}{r|rr}
$\rightarrow:$ & 0 & 1 \\
\hline 0 & 0 & 1 \\
1 & 1 & 1
\end{tabular}

\begin{tabular}{r|rr}
$\rightarrow:$ & 0 & 1 \\
\hline 0 & 1 & 1 \\
1 & 0 & 1
\end{tabular}

It is clear that $\mathbf{2}_{\mathbf{z}}, \mathbf{2}_{\mathbf{s}}, \mathbf{2}_{\mathbf{b}}$ are the only 2-element simple algebras in $\mathbf{I}$.

Recall, also, that the 3 -element (Kleene) algebra, namely $\mathbf{3}_{k}$, and the 4-element algebra, namely $\mathbf{4}_{d}$, are in DM. Their $\rightarrow$ operations are, respectively, as follows:

\begin{tabular}{r|rrr}
$\rightarrow:$ & 0 & 1 & 2 \\
\hline 0 & 1 & 1 & 1 \\
1 & 0 & 1 & 2 \\
2 & 2 & 1 & 2
\end{tabular}

\begin{tabular}{r|cccc}
$\rightarrow:$ & 0 & 1 & 2 & 3 \\
\hline 0 & 1 & 1 & 1 & 1 \\
1 & 0 & 1 & 2 & 3 \\
2 & 2 & 1 & 2 & 1 \\
3 & 3 & 1 & 1 & 3
\end{tabular}

and these are the only simple DM-algebras with more than 2 elements [1].

Hereinafter, we will focus on proving that if $\mathbf{A} \in \mathbf{I}$ is a simple algebra with $|A| \geq 3$ then $\mathbf{A} \in \mathbf{I}_{\mathbf{2}, \mathbf{0}}$.

Definition 5.1 Let $\mathbf{A} \in \mathbf{I}$. We define the relation $R^{\prime \prime}$ on $\mathbf{A}$ as follows:

$$
x R^{\prime \prime} y \text { if and only if } x^{\prime \prime}=y^{\prime \prime} .
$$

The following lemma is useful in proving that the relation $R^{\prime \prime}$ is a congruence on $\mathbf{A}$.

Lemma 5.2 [3, Lemma 3.4] Let $\mathbf{A}$ be an I-zroupoid. Then $\mathbf{A}$ satisfies: 


$$
(x \rightarrow y)^{\prime} \approx\left(x^{\prime \prime} \rightarrow y\right)^{\prime} .
$$

Lemma 5.3 Let $\mathbf{A} \in \mathbf{I}$. Then $R^{\prime \prime}$ is a congruence on $\mathbf{A}$.

Proof Clearly $R^{\prime \prime}$ is an equivalence relation. Let $x, y, z, t \in A$ with $x R^{\prime \prime} y$ and $z R^{\prime \prime} t$. Therefore, $x^{\prime \prime}=y^{\prime \prime}$ and $z^{\prime \prime}=t^{\prime \prime}$. Hence by [8, Lemma 7.5 (b)] and Lemma 5.2 we have that $(x \rightarrow z)^{\prime \prime}=(y \rightarrow t)^{\prime \prime}$. Consequently, $R^{\prime \prime}$ is a congruence on $\mathbf{A}$.

Lemma 5.4 Let $\mathbf{A} \in \mathbf{I}$. If $\mathbf{A} \models x^{\prime \prime} \approx y^{\prime \prime}$, then
(a) $\mathbf{A} \models x^{\prime \prime} \approx 0$
(b) $\mathbf{A} \models(x \rightarrow y)^{\prime} \approx 0$
(c) $\mathbf{A} \models(x \rightarrow y) \rightarrow z \approx 0$

\section{Proof}

(a) Let $a \in A$. Then $0=0^{\prime \prime}=a^{\prime \prime}$.

(b) Let $a, b \in A$. By (国) we have that $a^{\prime \prime}=b^{\prime \prime}=0$. Then, from [8, Lemma $\left.7.5(\mathrm{~b})\right],(a \rightarrow b)^{\prime}=$ $\left(a \rightarrow b^{\prime \prime}\right)^{\prime}=(a \rightarrow 0)^{\prime}=a^{\prime \prime}=0$.

(c) Let $a, b, c \in A$. Since (b) $), 0=\left[\left(c^{\prime} \rightarrow a\right) \rightarrow(b \rightarrow c)^{\prime}\right]^{\prime}=(a \rightarrow b) \rightarrow c$.

The proof of the following lemma is immediate.

Lemma 5.5 Let $\mathbf{A} \in \mathbf{I}$ satisfying the identity $(x \rightarrow y)^{\prime} \approx 0$ then the relation $R^{\prime}$ defined by,

$$
x R^{\prime} y \text { if and only if } x^{\prime}=y^{\prime},
$$

is a congruence on $\mathbf{A}$.

Theorem 5.6 Let $\mathbf{A} \in \mathbf{I}$ be a simple algebra with $|A| \geq 3$. Then $\mathbf{A} \in \mathbf{I}_{\mathbf{2}, \mathbf{0}}$.

Proof Assume that $\mathbf{A} \not \models x^{\prime \prime} \approx x$. Then there exist an element $a \in A$ with

(5.1) $a \neq a^{\prime \prime}$

Since $0=0 "$ we conclude that

(5.2) $a \neq 0$

By Lemma 5.3 we can affirm that the relation $R^{\prime \prime}$ (see Definition 5.1) is a congruence on $\mathbf{A}$. By [8, Corollary 7.7], we have $a^{\prime \prime \prime \prime}=a^{\prime \prime}$. Therefore, $a^{\prime \prime} R^{\prime \prime} a$. Since $\mathbf{A}$ is simple, and $a \neq a^{\prime \prime}$ by [5.1, we have

(5.3) $R^{\prime \prime}=A \times A$.

Hence $\mathbf{A} \models x^{\prime \prime} \approx y^{\prime \prime}$, which, by Lemma [5.4, implies

(5.4) $\mathbf{A} \models x^{\prime \prime} \approx 0$, 


$$
\mathbf{A} \models(x \rightarrow y)^{\prime} \approx 0
$$

and

$$
\mathbf{A} \models(x \rightarrow y) \rightarrow z \approx 0 .
$$

Observe from (5.4) that $a^{\prime \prime}=0=0^{\prime \prime}$, implying $a^{\prime} R^{\prime} 0^{\prime}$. Also, it follows from (5.5) and Lemma 5.5 that $R^{\prime}$ is a congruence.

If $a^{\prime} \neq 0^{\prime}$, since $\mathbf{A}$ is simple, we would have that $R^{\prime}=A \times A$. Then $0 R^{\prime} a$, and, consequently, $0^{\prime}=a^{\prime}$, which is a contradiction. Hence

(5.7) $a^{\prime}=0^{\prime}$.

By (5.7), $a R^{\prime} 0$ and, by (5.2),$R^{\prime}=A \times A$.

Let $b \in A$. Then $b R^{\prime} 0^{\prime}$. We can verify that $b^{\prime}=0^{\prime \prime}=0$. Consequently,

$$
\mathbf{A} \models x^{\prime} \approx 0 .
$$

From (5.8) we conclude that $\mathbf{A} \models 0^{\prime} \approx 0$. Then, for $b \in A$, we have $0 \rightarrow b=0^{\prime} \rightarrow b=(0 \rightarrow 0) \rightarrow$ $b=0$ by (5.6). Hence

$$
\mathbf{A} \models 0 \rightarrow x \approx 0 .
$$

Now we consider the following cases:

- Assume that $\mathbf{A} \models x \rightarrow y \approx 0$. Let $R_{a}$ denote the equivalence relation correspnding to the partition $\{\{0\}, A \backslash\{0\}\}$ of $A$. Notice that $|A \backslash\{0\}| \geq 2$ since $|A| \geq 3$, whence $R^{\prime}$ is nontrivial. Since $\mathbf{A} \models x \rightarrow y \approx 0, R_{a}$ is a congruence, which is a contradiction since the algebra is simple.

- Assume that $\mathbf{A} \not \models x \rightarrow y \approx 0$. Then the set

$$
T=\{b \in A: \quad \text { exists } c \in A \text { with } b \rightarrow c \neq 0\}
$$

is not empty, and from (5.9), $0 \notin T$. Then the equivalence relation $R_{T}$ associated to the partition $\{T, A \backslash T\}$ of $A$ satisfies that $R_{T} \neq \Delta$ and $R_{T} \neq A \times A$. Let the elements $b, c, d, e, f$ in $A$ with $b R_{T} c$ and $d R_{T} e$. Then, from (5.6), we have that $(b \rightarrow d) \rightarrow f=$ $(c \rightarrow e) \rightarrow f=0$. Hence $b \rightarrow d R_{T} c \rightarrow e$, implying that $R_{T}$ is a congruence on A. Since $R_{T} \neq \Delta$, we have a contradiction, because $\mathbf{A}$ is simple and $|A| \geq 3$.

Thus we have proved that $\mathbf{A} \models x^{\prime \prime} \approx x$, completing the proof.

In view of Theorem 4.5 and Theorem 5.6 we have the following crucial result.

Corollary 5.7 Let $\mathbf{A} \in \mathbf{I}$ be a simple algebra with $|A| \geq 3$. Then $\mathbf{A} \in \mathbf{D M}$.

Thus we have proved the following desired result describing the simple algebras of $\mathbf{I}$.

Theorem 5.8 The only simple algebras in $\mathbf{I}$ are $\mathbf{2}_{\mathbf{z}}, \mathbf{2}_{\mathrm{s}}, \mathbf{2}_{\mathrm{b}}, \mathbf{3}_{k}$ and $\mathbf{4}_{d}$.

Recall ([2]) that a variety is semisimple if and only if every subdirectly irreducible algebra in it is simple. 
Corollary 5.9 A subvariety $\mathbf{V}$ of $\mathbf{I}$ is semi-simple if and only if $\mathbf{V} \subseteq \mathbb{V}\left(\mathbf{2}_{\mathbf{z}}, \mathbf{2}_{\mathbf{s}}, \mathbf{4}_{d}\right)$.

Corollary 5.10 The lattice of semisimple subvarieties of $\mathbf{I}$ is isomorphic to the direct product of a 4-element Boolean lattice and and a 4-element chain and is the one depicted in the following figure, where $\mathbf{A}_{1}, \ldots, \mathbf{A}_{n}$ denotes $\mathbb{V}\left(\mathbf{A}_{1}, \ldots, \mathbf{A}_{n}\right)$.

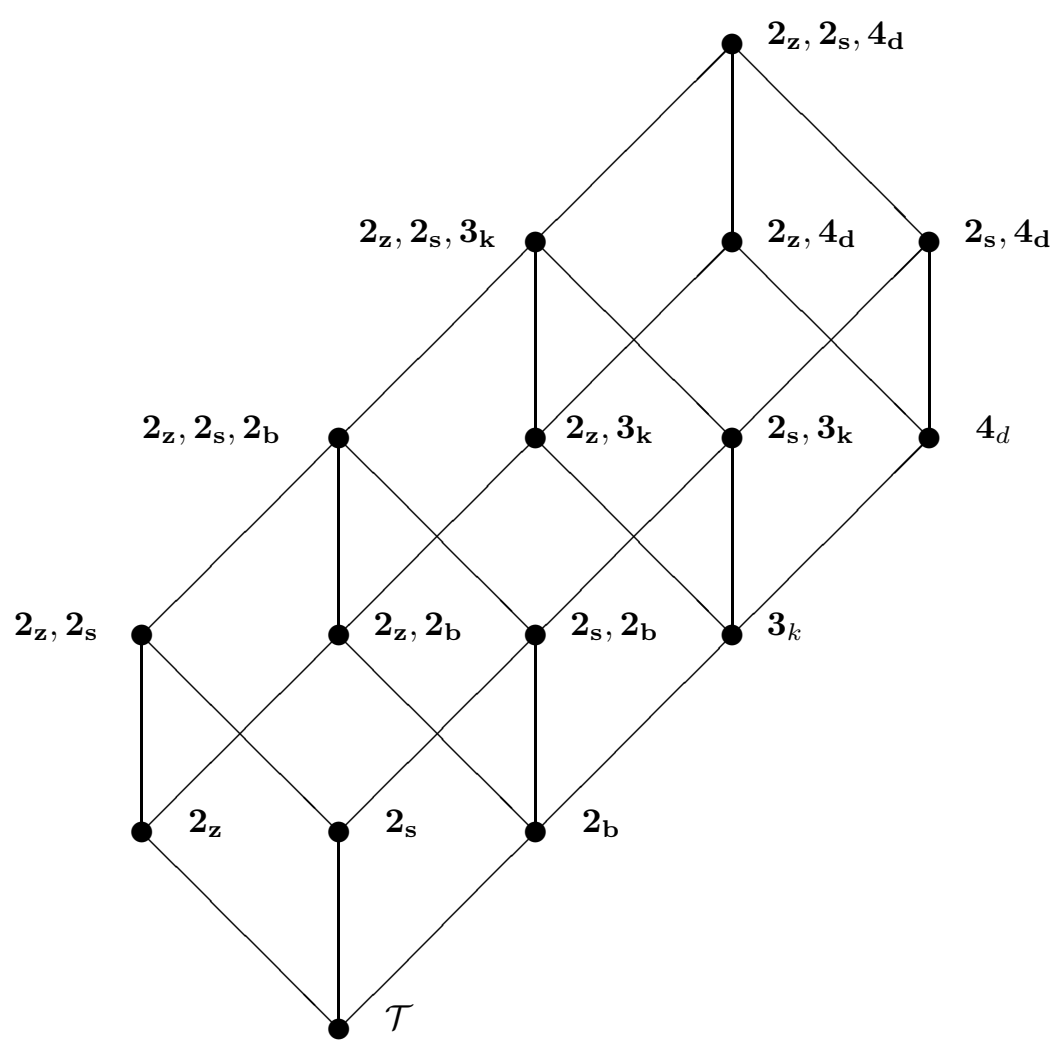

Corollary $\mathbf{5 . 1 1}$ Semisimple varieties of $\mathbf{I}$-zroupoids are locally finite.

We conclude this section by raising the problem of axiomatization of subvarieties of $\mathbb{V}\left(\mathbf{2}_{\mathbf{z}}, \mathbf{2}_{\mathbf{s}}, \mathbf{4}_{d}\right)$.

\section{Appendix}

Proof of Lemma 3.3 Items from (11) to (19) are proved in [3]. Let $a, b, c, d \in A$.

(201)

$$
\begin{array}{rlrl}
{[(0 \rightarrow a) \rightarrow b] \rightarrow a} & =\left[(b \rightarrow a) \rightarrow(0 \rightarrow a)^{\prime}\right]^{\prime} & \text { by (14) } \\
& =(b \rightarrow a)^{\prime \prime} & \text { by (15) using } x=b, y=a \\
& =b \rightarrow a & &
\end{array}
$$


(21)

$$
\begin{aligned}
a \rightarrow(0 \rightarrow b) & =[\{0 \rightarrow(0 \rightarrow b)\} \rightarrow a] \rightarrow(0 \rightarrow b) & & \text { by }(\underline{20}) \text { with } x=0 \rightarrow b, y=a \\
& =[(0 \rightarrow b) \rightarrow a] \rightarrow(0 \rightarrow b) & & \text { by }\left(\frac{77}{70}\right) \\
& =(0 \rightarrow a) \rightarrow(0 \rightarrow b) & & \text { by }(\underline{6})
\end{aligned}
$$

(22)

$$
\begin{array}{rlrl}
(a \rightarrow b)^{\prime} \rightarrow(0 \rightarrow c) & =[(a \rightarrow b) \rightarrow 0] \rightarrow(0 \rightarrow c) & \\
& =\left[\left\{(0 \rightarrow c)^{\prime} \rightarrow(a \rightarrow b)\right\} \rightarrow\{0 \rightarrow(0 \rightarrow c)\}^{\prime}\right]^{\prime} & & \text { by (I) } \\
& =\left[\left\{(0 \rightarrow c)^{\prime} \rightarrow(a \rightarrow b)\right\} \rightarrow(0 \rightarrow c)^{\prime}\right]^{\prime} & & \text { by (17) } \\
& =\left[\{0 \rightarrow(a \rightarrow b)\} \rightarrow(0 \rightarrow c)^{\prime}\right]^{\prime} & & \text { by (66) taking } x=a \rightarrow b, \\
& =\left[\{0 \rightarrow(a \rightarrow b)\} \rightarrow\left(c^{\prime} \rightarrow 0^{\prime}\right)^{\prime}\right]^{\prime} & & y=(0 \rightarrow c)^{\prime} \\
& =\left[(a \rightarrow b) \rightarrow c^{\prime}\right] \rightarrow 0^{\prime} & & \text { by Lemma 3.1 (国) } \\
& =0 \rightarrow\left[(a \rightarrow b) \rightarrow c^{\prime}\right]^{\prime} & & \text { from (I) } \\
& & & \text { by Lemma 3.1 (国) }
\end{array}
$$

(23)

$$
\begin{aligned}
{\left[\left(a^{\prime} \rightarrow b^{\prime}\right) \rightarrow(b \rightarrow a)^{\prime}\right] \rightarrow(b \rightarrow a) } & =\left[\left(a^{\prime} \rightarrow b^{\prime}\right) \rightarrow(b \rightarrow a)^{\prime}\right]^{\prime \prime} \rightarrow(b \rightarrow a) \\
& =\left[\left(b^{\prime} \rightarrow b\right) \rightarrow a\right]^{\prime} \rightarrow(b \rightarrow a) \\
& =[b \rightarrow a]^{\prime} \rightarrow(b \rightarrow a) \\
& =b \rightarrow a
\end{aligned}
$$

(24)

$$
\begin{aligned}
{\left[0 \rightarrow\left(a^{\prime} \rightarrow b^{\prime}\right)^{\prime}\right] \rightarrow(b \rightarrow a) } & =\left[\left(a^{\prime} \rightarrow b^{\prime}\right) \rightarrow 0^{\prime}\right] \rightarrow(b \rightarrow a) & & \text { by Lemma 3.1] (国) } \\
& =\left[\left(a^{\prime} \rightarrow b^{\prime}\right) \rightarrow(b \rightarrow a)^{\prime}\right] \rightarrow(b \rightarrow a) & & \text { by (11) } \\
& =b \rightarrow a & & \text { from (23) }
\end{aligned}
$$

(25)

$$
\begin{aligned}
(0 \rightarrow(a \rightarrow b)) \rightarrow a & =\left[\left(a^{\prime} \rightarrow 0\right) \rightarrow\{(a \rightarrow b) \rightarrow a\}^{\prime}\right]^{\prime} & & \text { from (I) } \\
& =\left[\left(a^{\prime} \rightarrow 0\right) \rightarrow\{(0 \rightarrow b) \rightarrow a\}^{\prime}\right]^{\prime} & & \text { by (6) } \\
& =[\{0 \rightarrow(0 \rightarrow b\}] \rightarrow a & & \text { using (I) } \\
& =(0 \rightarrow b) \rightarrow a & & \text { by (17) }
\end{aligned}
$$

(26)

$$
\begin{array}{rlrl}
(0 \rightarrow a) \rightarrow(a \rightarrow b) & =[(a \rightarrow b) \rightarrow a] \rightarrow(a \rightarrow b) & & \text { by (6) with } y=a \rightarrow b, x=a \\
& =\left[a \rightarrow(b \rightarrow a)^{\prime}\right]^{\prime} \rightarrow(a \rightarrow b) & & \text { by (15) } \\
& =\left[a^{\prime \prime} \rightarrow(b \rightarrow a)^{\prime}\right]^{\prime} \rightarrow(a \rightarrow b) & & \\
& =\left[\left(a^{\prime} \rightarrow 0\right) \rightarrow(b \rightarrow a)^{\prime}\right]^{\prime} \rightarrow(a \rightarrow b) & \\
& =[(0 \rightarrow b) \rightarrow a] \rightarrow(a \rightarrow b) & & \text { from (I) } \\
& =[\{0 \rightarrow(a \rightarrow b)\} \rightarrow a] \rightarrow(a \rightarrow b) & & \text { by (25) } \\
& =a \rightarrow(a \rightarrow b) & & \text { by (201) with } x=a \rightarrow b, y=a
\end{array}
$$

(27)

$$
\begin{array}{rlr}
{[b \rightarrow(0 \rightarrow a)]^{\prime}} & =[(0 \rightarrow b) \rightarrow(0 \rightarrow a)]^{\prime} & \text { by (21) } \\
& =\left[\left\{(0 \rightarrow a)^{\prime} \rightarrow 0\right\} \rightarrow\{b \rightarrow(0 \rightarrow a)\}^{\prime}\right]^{\prime \prime} & \text { from (I) } \\
& =\left[(0 \rightarrow a)^{\prime} \rightarrow 0\right] \rightarrow[b \rightarrow(0 \rightarrow a)]^{\prime} & \\
& =(0 \rightarrow a)^{\prime \prime} \rightarrow[b \rightarrow(0 \rightarrow a)]^{\prime} & \\
& =(0 \rightarrow a) \rightarrow[b \rightarrow(0 \rightarrow a)]^{\prime} &
\end{array}
$$


(28)

$$
\begin{aligned}
{[(a \rightarrow b) \rightarrow(0 \rightarrow a)]^{\prime} } & =\left[\left\{(0 \rightarrow a)^{\prime} \rightarrow a\right\} \rightarrow\{b \rightarrow(0 \rightarrow a)\}^{\prime}\right]^{\prime \prime} & \text { from (I) } \\
& =\left[(0 \rightarrow a)^{\prime} \rightarrow a\right] \rightarrow[b \rightarrow(0 \rightarrow a)]^{\prime} & \\
& =[\{(0 \rightarrow a) \rightarrow 0\} \rightarrow a] \rightarrow[b \rightarrow(0 \rightarrow a)]^{\prime} & \\
& =(0 \rightarrow a) \rightarrow[b \rightarrow(0 \rightarrow a)]^{\prime} & \text { by (20) with } x=a, y=0
\end{aligned}
$$

(29)

$$
\begin{array}{rlr}
b \rightarrow(0 \rightarrow a) & =[b \rightarrow(0 \rightarrow a)]^{\prime \prime} & \\
& =\left[(0 \rightarrow a) \rightarrow\{b \rightarrow(0 \rightarrow a)\}^{\prime}\right]^{\prime} & \text { by }(\underline{27}) \\
& =[(a \rightarrow b) \rightarrow(0 \rightarrow a)]^{\prime \prime} & \text { by }(\underline{28}) \\
& =(a \rightarrow b) \rightarrow(0 \rightarrow a) . &
\end{array}
$$

(30)

$$
\begin{aligned}
b \rightarrow(b \rightarrow a) & =(0 \rightarrow b) \rightarrow(b \rightarrow a) & & \text { by }(\underline{26}) \\
& =[0 \rightarrow(0 \rightarrow b)] \rightarrow(b \rightarrow a) & & \text { by (7) } \\
& =[(b \rightarrow a) \rightarrow(0 \rightarrow b)] \rightarrow(b \rightarrow a) & & \text { by }(\underline{6}) \\
& =[a \rightarrow(0 \rightarrow b)] \rightarrow(b \rightarrow a) & & \text { by }(29)
\end{aligned}
$$

(31)

$$
\begin{aligned}
& a \rightarrow b=\left[0 \rightarrow\left(b^{\prime} \rightarrow a^{\prime}\right)^{\prime}\right] \rightarrow(a \rightarrow b) \quad \text { by (24) } \\
& =\left[0 \rightarrow\left\{(b \rightarrow 0) \rightarrow a^{\prime}\right\}^{\prime}\right] \rightarrow(a \rightarrow b) \\
& =\left[(b \rightarrow 0)^{\prime} \rightarrow(0 \rightarrow a)\right] \rightarrow(a \rightarrow b) \text { by (22) } \\
& =\left[b^{\prime \prime} \rightarrow(0 \rightarrow a)\right] \rightarrow(a \rightarrow b) \\
& =[b \rightarrow(0 \rightarrow a)] \rightarrow(a \rightarrow b) \\
& =a \rightarrow(a \rightarrow b) \\
& \text { by }(30)
\end{aligned}
$$

(32)

$$
\begin{aligned}
c \rightarrow((a \rightarrow b) \rightarrow c)^{\prime} & =[\{c \rightarrow(a \rightarrow b)\} \rightarrow c]^{\prime} \text { by }(15) \\
& =[\{0 \rightarrow(a \rightarrow b)\} \rightarrow c]^{\prime} \text { by (6) } \\
& =[\{a \rightarrow(0 \rightarrow b)\} \rightarrow c]^{\prime} \text { by }(13)
\end{aligned}
$$

(33)

$$
\begin{aligned}
& {\left[0 \rightarrow(a \rightarrow b)^{\prime}\right] \rightarrow b=\left[(a \rightarrow b) \rightarrow 0^{\prime}\right] \rightarrow b \quad \text { by Lemma } 3.1 \text { (国) }} \\
& =\left[(a \rightarrow b) \rightarrow b^{\prime}\right] \rightarrow b \text { by (1) } \\
& =\left[\left(a \rightarrow 0^{\prime}\right) \rightarrow b^{\prime}\right] \rightarrow b \text { by (1) } \\
& =\left[\left(a \rightarrow 0^{\prime}\right) \rightarrow 0^{\prime}\right] \rightarrow b \text { by (1) } \\
& =\left[\left(0 \rightarrow a^{\prime}\right) \rightarrow 0^{\prime}\right] \rightarrow b \text { by Lemma } 3.1 \text { (a) } \\
& =\left[0 \rightarrow\left(0 \rightarrow a^{\prime}\right)^{\prime}\right] \rightarrow b \text { by Lemma } 3.1 \text { (国) } \\
& =\left(0 \rightarrow a^{\prime \prime}\right) \rightarrow b \quad \text { by (11) } \\
& =(0 \rightarrow a) \rightarrow b
\end{aligned}
$$

(34)

$$
\begin{array}{rlrl}
{\left[\left\{a \rightarrow\left(0 \rightarrow(b \rightarrow c)^{\prime}\right)\right\} \rightarrow c\right]^{\prime}} & =\left(c^{\prime} \rightarrow a\right) \rightarrow\left[\left\{0 \rightarrow(b \rightarrow c)^{\prime}\right\} \rightarrow c\right]^{\prime} & & \text { by }(\mathrm{I}) \\
& =\left(c^{\prime} \rightarrow a\right) \rightarrow[(0 \rightarrow b) \rightarrow c]^{\prime} & & \text { by (133) } \\
& =\left(c^{\prime} \rightarrow a\right) \rightarrow[(c \rightarrow b) \rightarrow c]^{\prime} & & \text { by (16) } \\
& =\left(c^{\prime} \rightarrow a\right) \rightarrow\left[c \rightarrow(b \rightarrow c)^{\prime}\right]^{\prime \prime} & & \text { by (15) } \\
& =\left(c^{\prime} \rightarrow a\right) \rightarrow\left[c \rightarrow(b \rightarrow c)^{\prime}\right] &
\end{array}
$$


(35)

$$
\begin{aligned}
& a \rightarrow\left[\left\{b \rightarrow(c \rightarrow a)^{\prime}\right\} \rightarrow a\right]^{\prime}=\left[\left\{b \rightarrow\left(0 \rightarrow(c \rightarrow a)^{\prime}\right)\right\} \rightarrow a\right]^{\prime} \quad \text { by (32) with } x=b, y=(c \rightarrow a)^{\prime}, \\
& z=a \\
& =\left(a^{\prime} \rightarrow b\right) \rightarrow\left\{a \rightarrow(c \rightarrow a)^{\prime}\right\} \quad \text { by (34) with } x=b, y=c, z=a
\end{aligned}
$$

(36)

$$
\begin{aligned}
\{0 \rightarrow(a \rightarrow b)\} \rightarrow b^{\prime} & =\left[(b \rightarrow 0) \rightarrow\left\{(a \rightarrow b) \rightarrow b^{\prime}\right\}^{\prime}\right]^{\prime} & & \text { from (I) } \\
& =\left[(b \rightarrow 0) \rightarrow\left\{\left(a \rightarrow 0^{\prime}\right) \rightarrow b^{\prime}\right\}^{\prime}\right]^{\prime} & & \text { by (11) } \\
& =\left\{0 \rightarrow\left(a \rightarrow 0^{\prime}\right)\right\} \rightarrow b^{\prime} & & \text { from (I) } \\
& =\left\{0 \rightarrow\left(0 \rightarrow a^{\prime}\right)\right\} \rightarrow b^{\prime} & & \text { by Lemma 3.1 (国) } \\
& =\left(0 \rightarrow a^{\prime}\right) \rightarrow b^{\prime} & & \text { by (17) } \\
& =\left(a \rightarrow 0^{\prime}\right) \rightarrow b^{\prime} & & \text { by Lemma 3.1 (国) } \\
& =\left(a \rightarrow b^{\prime \prime}\right) \rightarrow b^{\prime} & & \text { by (11) } \\
& =(a \rightarrow b) \rightarrow b^{\prime} & & \\
& =b \rightarrow(a \rightarrow b)^{\prime} & & \text { by (17) }
\end{aligned}
$$

(37)

$$
\begin{array}{rlrl}
\left(a^{\prime} \rightarrow b\right) \rightarrow\left\{a \rightarrow(c \rightarrow a)^{\prime}\right\} & =a \rightarrow\left[\left\{b \rightarrow(c \rightarrow a)^{\prime}\right\} \rightarrow a\right]^{\prime} & & \text { by (135) } \\
& =(0 \rightarrow b) \rightarrow\left[\{0 \rightarrow(c \rightarrow a)\} \rightarrow a^{\prime}\right] & & \text { by }(\underline{16}) \text { with } x=a, y=b, \\
& & z=c \rightarrow a
\end{array}
$$

(38)

$$
\begin{array}{rlrl}
a \rightarrow[(c \rightarrow b) \rightarrow a]^{\prime} & =[\{c \rightarrow(0 \rightarrow b)\} \rightarrow a]^{\prime} & & \text { by }(\underline{32}) \text { with } x=c, y=b, z=a \\
& =[\{(b \rightarrow c) \rightarrow(0 \rightarrow b)\} \rightarrow a]^{\prime} & & \text { by }(\underline{(29)}) \\
& =\left(a^{\prime} \rightarrow(b \rightarrow c)\right) \rightarrow[(0 \rightarrow b) \rightarrow a]^{\prime} & & \text { by }(\mathrm{I}) \\
& =\left\{a^{\prime} \rightarrow(b \rightarrow c)\right\} \rightarrow[(a \rightarrow b) \rightarrow a]^{\prime} & & \text { by (6) } \\
& =\left\{a^{\prime} \rightarrow(b \rightarrow c)\right\} \rightarrow\left[a \rightarrow(b \rightarrow a)^{\prime}\right]^{\prime \prime} & & \text { by (15) } \\
& =\left\{a^{\prime} \rightarrow(b \rightarrow c)\right\} \rightarrow\left[a \rightarrow(b \rightarrow a)^{\prime}\right] &
\end{array}
$$

(39)

$$
\begin{aligned}
a \rightarrow[(b \rightarrow c) \rightarrow a]^{\prime} & =\left[a^{\prime} \rightarrow(c \rightarrow b)\right] \rightarrow\left[a \rightarrow(c \rightarrow a)^{\prime}\right] \quad \text { by (138) with } x=a, y=c, z=b \\
& =[0 \rightarrow(c \rightarrow b)] \rightarrow\left[a \rightarrow(c \rightarrow a)^{\prime}\right] \quad \text { by (137) with } x=a, y=c \rightarrow b, z=c
\end{aligned}
$$

(40)

$$
\begin{array}{rlrl}
{[0 \rightarrow(a \rightarrow b)] \rightarrow\left[c \rightarrow(a \rightarrow c)^{\prime}\right]} & =c \rightarrow[(b \rightarrow a) \rightarrow c]^{\prime} & & \text { by (39) with } x=c, y=b, z=a \\
& =[\{c \rightarrow(b \rightarrow a)\} \rightarrow c]^{\prime} & & \text { by (15) } \\
& =[\{0 \rightarrow(b \rightarrow a)\} \rightarrow c]^{\prime} & & \text { by (6) } \\
& =[\{b \rightarrow(0 \rightarrow a)\} \rightarrow c]^{\prime} & & \text { by (13) } \\
& =\left(c^{\prime} \rightarrow b\right) \rightarrow[(0 \rightarrow a) \rightarrow c]^{\prime} & \text { from (I) } \\
& =\left(c^{\prime} \rightarrow b\right) \rightarrow[(c \rightarrow a) \rightarrow c]^{\prime} & \text { by (16) } \\
& =\left(c^{\prime} \rightarrow b\right) \rightarrow\left[c \rightarrow(a \rightarrow c)^{\prime}\right] & \text { by (15) } \\
& =(0 \rightarrow b) \rightarrow\left[c \rightarrow(a \rightarrow c)^{\prime}\right] & \text { by (137) with } x=c, y=b, z=a
\end{array}
$$


(41)

$$
\begin{aligned}
a \rightarrow[(b \rightarrow c) \rightarrow a]^{\prime} & =[0 \rightarrow(c \rightarrow b)] \rightarrow\left[a \rightarrow(c \rightarrow a)^{\prime}\right] & \text { by (39) } \\
& =(0 \rightarrow b) \rightarrow\left[a \rightarrow(c \rightarrow a)^{\prime}\right] & \text { by (40) with } x=c, y=b, z=a
\end{aligned}
$$

(42) This follows immediately from (18).

(43)

$$
\begin{array}{rll}
a \rightarrow(b \rightarrow a)^{\prime} & =[(a \rightarrow b) \rightarrow a]^{\prime} \quad \text { by }(15) \\
& =[(0 \rightarrow b) \rightarrow a]^{\prime} \quad \text { by (16) } \\
& =\left[\left(b^{\prime} \rightarrow 0^{\prime}\right) \rightarrow a\right]^{\prime} \quad \text { by Lemma } 3.1 \text { (国) } \\
& =\left(0 \rightarrow b^{\prime}\right) \rightarrow a^{\prime} \quad \text { by (19) } \\
& =\left(b \rightarrow 0^{\prime}\right) \rightarrow a^{\prime} \quad \text { by Lemma } 3.1 \text { (国) }
\end{array}
$$

(44)

$$
\begin{array}{rlrl}
\left(a^{\prime} \rightarrow b\right) \rightarrow a^{\prime} & =\left[a^{\prime} \rightarrow\left(b \rightarrow a^{\prime}\right)^{\prime}\right]^{\prime} & \text { by }(\underline{5}) \\
& =\left[\left(b \rightarrow 0^{\prime}\right) \rightarrow a^{\prime \prime}\right]^{\prime} & \text { by }(\underline{43}) \\
& =\left[\left(b \rightarrow 0^{\prime}\right) \rightarrow a\right]^{\prime} & \\
& =\left[\left(0 \rightarrow b^{\prime}\right) \rightarrow a\right]^{\prime} & & \text { by Lemma }[3.1(\text { b }) \\
& =\left[\left(a \rightarrow b^{\prime}\right) \rightarrow a\right]^{\prime} & & \text { by (6) } \\
& =a^{\prime} & & \text { by Hyphotesis }
\end{array}
$$

(45)

$$
\begin{aligned}
{\left[\{b \rightarrow(0 \rightarrow c)\} \rightarrow\left(a \rightarrow 0^{\prime}\right)^{\prime}\right]^{\prime} } & =\left[\left\{\left(a \rightarrow 0^{\prime}\right)^{\prime \prime} \rightarrow b\right\} \rightarrow\left\{(0 \rightarrow c) \rightarrow\left(a \rightarrow 0^{\prime}\right)^{\prime}\right\}^{\prime}\right]^{\prime \prime} & & \text { by (I) } \\
& =\left[\left(a \rightarrow 0^{\prime}\right) \rightarrow b\right] \rightarrow\left[(0 \rightarrow c) \rightarrow\left(a \rightarrow 0^{\prime}\right)^{\prime}\right]^{\prime} & & \\
& =\left[\left(a \rightarrow 0^{\prime}\right) \rightarrow b\right] \rightarrow\left[(c \rightarrow a) \rightarrow 0^{\prime}\right] & & \text { by (I) } \\
& =\left[\left(a \rightarrow 0^{\prime}\right) \rightarrow b\right] \rightarrow\left[0 \rightarrow(c \rightarrow a)^{\prime}\right] & & \text { by Lemma 3.1 (b) (b) } \\
& =\left[\left(0 \rightarrow a^{\prime} \rightarrow b\right] \rightarrow\left[0 \rightarrow(c \rightarrow a)^{\prime}\right]\right. & & \text { by Lemma 3.1 (b) (b) }
\end{aligned}
$$

(46)

$$
\begin{array}{rlrl}
{[\{a \rightarrow(0 \rightarrow b)\} \rightarrow c]^{\prime}} & =\left[\left(c^{\prime} \rightarrow a\right) \rightarrow\{(0 \rightarrow b) \rightarrow c\}^{\prime}\right]^{\prime \prime} & \text { by }(\mathrm{I}) \\
& =\left(c^{\prime} \rightarrow a\right) \rightarrow[(0 \rightarrow b) \rightarrow c]^{\prime} & \\
& =\left(c^{\prime} \rightarrow a\right) \rightarrow[(c \rightarrow b) \rightarrow c]^{\prime} & \text { by ([6) } \\
& =\left(c^{\prime} \rightarrow a\right) \rightarrow\left[c \rightarrow(b \rightarrow c)^{\prime}\right]^{\prime \prime} & \text { by (15) } \\
& =\left(c^{\prime} \rightarrow a\right) \rightarrow\left[c \rightarrow(b \rightarrow c)^{\prime}\right] & \\
& =(0 \rightarrow a) \rightarrow\left[c \rightarrow(b \rightarrow c)^{\prime}\right] & & \text { by (37) }
\end{array}
$$

(47)

$$
\begin{aligned}
{\left[\left(0 \rightarrow a^{\prime}\right) \rightarrow b\right] \rightarrow\left[0 \rightarrow(c \rightarrow a)^{\prime}\right] } & =\left[\{b \rightarrow(0 \rightarrow c)\} \rightarrow\left(a \rightarrow 0^{\prime}\right)^{\prime}\right]^{\prime} & & \text { by (45) } \\
& =(0 \rightarrow b) \rightarrow\left[\left(a \rightarrow 0^{\prime}\right)^{\prime} \rightarrow\left\{c \rightarrow\left(a \rightarrow 0^{\prime}\right)^{\prime}\right\}^{\prime}\right] & & \text { by (46) with } \\
& & & x=b, y=c, \\
& =(0 \rightarrow b) \rightarrow\left[\left(a \rightarrow 0^{\prime}\right)^{\prime} \rightarrow\left\{c \rightarrow\left(a \rightarrow 0^{\prime}\right)^{\prime}\right\}^{\prime}\right]^{\prime \prime} & & z=\left(a \rightarrow 0^{\prime}\right)^{\prime} \\
& \left.=(0 \rightarrow b) \rightarrow\left[\left(a \rightarrow 0^{\prime}\right)^{\prime} \rightarrow c\right\} \rightarrow\left(a \rightarrow 0^{\prime}\right)^{\prime}\right]^{\prime} & & \text { by (15) } \\
& =(0 \rightarrow b) \rightarrow\left[(0 \rightarrow c) \rightarrow\left(a \rightarrow 0^{\prime}\right)^{\prime}\right]^{\prime} & & \text { by (6) } \\
& =(0 \rightarrow b) \rightarrow\left[(c \rightarrow a) \rightarrow 0^{\prime}\right] & & \text { by (I) } \\
& =(0 \rightarrow b) \rightarrow\left[0 \rightarrow(c \rightarrow a)^{\prime}\right] & & \text { by Lemma 3.1 (b) (b) } \\
& =b \rightarrow\left[0 \rightarrow(c \rightarrow a)^{\prime}\right] & & \text { by (21) }
\end{aligned}
$$


(48)

$$
\begin{array}{rlrl}
{[(0 \rightarrow a) \rightarrow b] \rightarrow(0 \rightarrow c)} & =[0 \rightarrow\{(0 \rightarrow a) \rightarrow b\}] \rightarrow(0 \rightarrow c) & \text { by (21) } \\
& =[(0 \rightarrow a \rightarrow(0 \rightarrow b)] \rightarrow(0 \rightarrow c) & \text { by (13) } \\
& =[a \rightarrow(0 \rightarrow b)] \rightarrow(0 \rightarrow c) & & \text { by (21) } \\
& =[0 \rightarrow(a \rightarrow b)] \rightarrow(0 \rightarrow c) & & \text { by (13) } \\
& =(a \rightarrow b) \rightarrow(0 \rightarrow c) & & \text { by (21) } \\
& =0 \rightarrow((a \rightarrow b) \rightarrow c) & & \text { by (13) }
\end{array}
$$

(49)

$$
\begin{aligned}
b \rightarrow\left[0 \rightarrow(c \rightarrow a)^{\prime}\right] & =\left[\left(0 \rightarrow a^{\prime}\right) \rightarrow b\right] \rightarrow\left[0 \rightarrow(c \rightarrow a)^{\prime}\right] & & \text { by (47) } \\
& =0 \rightarrow\left[\left(a^{\prime} \rightarrow b\right) \rightarrow(c \rightarrow a)^{\prime}\right] & & \text { by (48) } \\
& =\left(a^{\prime} \rightarrow b\right) \rightarrow\left[0 \rightarrow(c \rightarrow a)^{\prime}\right] & & \text { by (13) }
\end{aligned}
$$

(50)

$$
\begin{aligned}
b \rightarrow\left[0 \rightarrow(c \rightarrow a)^{\prime}\right] & =\left(a^{\prime} \rightarrow b\right) \rightarrow\left[0 \rightarrow(c \rightarrow a)^{\prime}\right] \quad \text { by }(149) \\
& =0 \rightarrow\left[\left(a^{\prime} \rightarrow b\right) \rightarrow(c \rightarrow a)^{\prime}\right] \quad \text { by }
\end{aligned}
$$

(51)

$$
\begin{aligned}
& (0 \rightarrow a) \rightarrow\left[\{0 \rightarrow(b \rightarrow c)\} \rightarrow d^{\prime}\right]=(0 \rightarrow a) \rightarrow\left[\{0 \rightarrow(b \rightarrow c)\} \rightarrow\left\{d \rightarrow(0 \rightarrow d)^{\prime}\right\}\right] \text { by (3) } \\
& =(0 \rightarrow a) \rightarrow\left[d \rightarrow\left[(b \rightarrow c)^{\prime} \rightarrow d\right]\right]^{\prime} \quad \text { by (41) with } \\
& =\left[\left\{a \rightarrow\left(0 \rightarrow(b \rightarrow c)^{\prime}\right)\right\} \rightarrow d\right]^{\prime} \\
& =\left[\left[0 \rightarrow\left\{\left(c^{\prime} \rightarrow a\right) \rightarrow(b \rightarrow c)^{\prime}\right\}\right] \rightarrow d\right]^{\prime} \\
& =\left[\left[0 \rightarrow\left\{\left(c^{\prime} \rightarrow a\right) \rightarrow(b \rightarrow c)^{\prime}\right\}^{\prime \prime}\right] \rightarrow d\right]^{\prime} \\
& =\left[\left[0 \rightarrow\{(a \rightarrow b) \rightarrow c\}^{\prime}\right] \rightarrow d\right]^{\prime} \\
& =\left[\left[d \rightarrow[(a \rightarrow b) \rightarrow c]^{\prime}\right] \rightarrow d\right]^{\prime} \\
& =\left[d \rightarrow\left\{((a \rightarrow b) \rightarrow c)^{\prime} \rightarrow d\right\}^{\prime}\right]^{\prime \prime} \\
& =d \rightarrow\left[\{(a \rightarrow b) \rightarrow c\}^{\prime} \rightarrow d\right]^{\prime} \\
& =[0 \rightarrow\{(a \rightarrow b) \rightarrow c\}] \rightarrow\left[d \rightarrow(0 \rightarrow d)^{\prime}\right] \\
& =[0 \rightarrow\{(a \rightarrow b) \rightarrow c\}] \rightarrow d^{\prime} \\
& x=d, y=b \rightarrow c \text {, } \\
& z=0
\end{aligned}
$$

(52)

$$
\begin{array}{rlrl}
(0 \rightarrow a) \rightarrow\left[\left(0 \rightarrow b^{\prime}\right) \rightarrow(0 \rightarrow c)^{\prime}\right] & =(0 \rightarrow a) \rightarrow\left[\{0 \rightarrow(b \rightarrow 0)\} \rightarrow(0 \rightarrow c)^{\prime}\right] & \\
& =[0 \rightarrow\{(a \rightarrow b) \rightarrow 0\}] \rightarrow(0 \rightarrow c)^{\prime} & & \text { by (51) with } \\
& & & x=a, y=b, z=0, \\
& =\left[0 \rightarrow(a \rightarrow b)^{\prime}\right] \rightarrow(0 \rightarrow c)^{\prime} & & u=0 \rightarrow c \\
& =[(a \rightarrow b) \rightarrow(0 \rightarrow c)]^{\prime} & & \\
& =[0 \rightarrow\{(a \rightarrow b) \rightarrow c\}]^{\prime} & & \text { by (10) }
\end{array}
$$


(53)

$$
\begin{aligned}
& 0 \rightarrow\left[(a \rightarrow b) \rightarrow\left(c \rightarrow a^{\prime}\right)^{\prime}\right]=0 \rightarrow\left[\left(a^{\prime \prime} \rightarrow b\right) \rightarrow\left(c \rightarrow a^{\prime}\right)^{\prime}\right]^{\prime \prime} \\
& =0 \rightarrow\left[(b \rightarrow c) \rightarrow a^{\prime}\right]^{\prime} \quad \text { by }(\mathrm{I}) \\
& =0 \rightarrow\left[0 \rightarrow\left\{(b \rightarrow c) \rightarrow a^{\prime}\right\}^{\prime}\right] \quad \text { by (7) } \\
& =0 \rightarrow\left[(0 \rightarrow b) \rightarrow\left\{\left(0 \rightarrow c^{\prime}\right) \rightarrow\left(0 \rightarrow a^{\prime}\right)^{\prime}\right\}\right] \text { by (152) with } \\
& =0 \rightarrow\left[(0 \rightarrow b) \rightarrow\left\{\left(0 \rightarrow c^{\prime}\right) \rightarrow\left(a \rightarrow 0^{\prime}\right)^{\prime}\right\}\right] \text { by Lemma } 3.1 \text { (b) } \\
& =0 \rightarrow\left[(0 \rightarrow b) \rightarrow\left\{\left(0 \rightarrow c^{\prime}\right) \rightarrow\left(a \rightarrow 0^{\prime}\right)^{\prime}\right\}^{\prime \prime}\right] \\
& =0 \rightarrow\left[(0 \rightarrow b) \rightarrow\left\{\left(c^{\prime} \rightarrow a\right) \rightarrow 0^{\prime}\right\}^{\prime}\right] \quad \text { by }(\mathrm{I}) \\
& =0 \rightarrow\left[(0 \rightarrow b) \rightarrow\left\{\left(c^{\prime} \rightarrow a\right) \rightarrow 0^{\prime}\right\}^{\prime}\right]^{\prime \prime} \\
& =0 \rightarrow\left[\left\{b \rightarrow\left(c^{\prime} \rightarrow a\right)\right\} \rightarrow 0^{\prime}\right]^{\prime} \\
& =0 \rightarrow\left[0 \rightarrow\left\{b \rightarrow\left(c^{\prime} \rightarrow a\right)\right\}^{\prime}\right]^{\prime} \\
& =0 \rightarrow\left[b \rightarrow\left(c^{\prime} \rightarrow a\right)\right]^{\prime \prime} \\
& =0 \rightarrow\left[b \rightarrow\left(c^{\prime} \rightarrow a\right)\right]
\end{aligned}
$$

(54)

$$
\begin{array}{rlrl}
0 \rightarrow\left[(a \rightarrow b)^{\prime} \rightarrow c\right] & =0 \rightarrow\left[(a \rightarrow b) \rightarrow c^{\prime}\right]^{\prime} & \text { by (8) } \\
& =0 \rightarrow\left[\left(c^{\prime \prime} \rightarrow a\right) \rightarrow\left(b \rightarrow c^{\prime}\right)^{\prime}\right]^{\prime \prime} & & \text { by }(\mathrm{I}) \\
& =0 \rightarrow\left[(c \rightarrow a) \rightarrow\left(b \rightarrow c^{\prime}\right)^{\prime}\right] & & \\
& =0 \rightarrow\left[a \rightarrow\left(b^{\prime} \rightarrow c\right)\right] & & \text { by (153) }
\end{array}
$$

(55)

$$
\begin{aligned}
{\left[\{(a \rightarrow b) \rightarrow c\} \rightarrow\{0 \rightarrow(b \rightarrow c)\}^{\prime}\right] \rightarrow\{(a \rightarrow b) \rightarrow c\} } & =\left[0 \rightarrow\{0 \rightarrow(b \rightarrow c)\}^{\prime}\right] \rightarrow\{(a \rightarrow b) \rightarrow c\} & \text { by (6) } \\
& =\left[0 \rightarrow(b \rightarrow c)^{\prime}\right] \rightarrow[(a \rightarrow b) \rightarrow c] & \text { by (ㄱ) } \\
& =\left[0 \rightarrow(b \rightarrow c)^{\prime}\right] \rightarrow\left[\left(c^{\prime} \rightarrow a\right) \rightarrow(b \rightarrow c)^{\prime}\right]^{\prime} & \text { by (I) } \\
& =\left[\left(c^{\prime} \rightarrow a\right) \rightarrow(b \rightarrow c)^{\prime}\right]^{\prime} & \text { by (44) } \\
& =(a \rightarrow b) \rightarrow c & \text { by (I) }
\end{aligned}
$$

(56)

$$
\begin{array}{rlrl}
{\left[0 \rightarrow(a \rightarrow b)^{\prime}\right] \rightarrow(c \rightarrow b)} & =\left[0 \rightarrow\left(a^{\prime \prime} \rightarrow b\right)^{\prime}\right] \rightarrow(c \rightarrow b) & \\
& =\left[a^{\prime} \rightarrow\left(0 \rightarrow b^{\prime}\right)\right] \rightarrow(c \rightarrow b) & & \text { by (12) } \\
& =\left[0 \rightarrow\left\{\left(0 \rightarrow a^{\prime}\right) \rightarrow b^{\prime}\right\}\right] \rightarrow(c \rightarrow b) & & \text { by (42) } \\
& =\left[\left(0 \rightarrow a^{\prime}\right) \rightarrow\left(0 \rightarrow b^{\prime}\right)\right] \rightarrow(c \rightarrow b) & & \text { by (13) } \\
& =\left[\left\{(c \rightarrow b)^{\prime} \rightarrow\left(0 \rightarrow a^{\prime}\right)\right\} \rightarrow\left\{\left(0 \rightarrow b^{\prime}\right) \rightarrow(c \rightarrow b)\right\}^{\prime}\right]^{\prime} & & \text { by (I) } \\
& =\left[\left\{(c \rightarrow b)^{\prime} \rightarrow\left(0 \rightarrow a^{\prime}\right)\right\} \rightarrow(c \rightarrow b)^{\prime}\right]^{\prime} & & \text { by (2) } \\
& =\left[(c \rightarrow b)^{\prime} \rightarrow\left\{\left(0 \rightarrow a^{\prime}\right) \rightarrow(c \rightarrow b)^{\prime}\right\}^{\prime}\right]^{\prime \prime} & & \text { by (15) } \\
& =(c \rightarrow b)^{\prime} \rightarrow\left[\left(0 \rightarrow a^{\prime}\right) \rightarrow(c \rightarrow b)^{\prime}\right]^{\prime} & & \\
& =\left[\left(0 \rightarrow a^{\prime}\right) \rightarrow 0^{\prime}\right] \rightarrow(c \rightarrow b)^{\prime \prime} & & \text { by (43) } \\
& =\left[\left(0 \rightarrow a^{\prime}\right) \rightarrow 0^{\prime}\right] \rightarrow(c \rightarrow b) & & \text { by Lemma 3.1] (b) } \\
& =\left[0 \rightarrow\left(0 \rightarrow a^{\prime}\right)^{\prime}\right] \rightarrow(c \rightarrow b) & & \text { by (11) } \\
& =\left(0 \rightarrow a^{\prime \prime}\right) \rightarrow(c \rightarrow b) &
\end{array}
$$

(57)

$$
\begin{aligned}
{\left[b \rightarrow\left[0 \rightarrow(0 \rightarrow c)^{\prime}\right]\right] \rightarrow a } & =\left[b \rightarrow\left(0 \rightarrow c^{\prime}\right)\right] \rightarrow a \text { by }(111) \\
& =\left[0 \rightarrow\left(b^{\prime} \rightarrow c\right)^{\prime}\right] \rightarrow a \text { by (12) } \\
& =\left[a \rightarrow\left(b^{\prime} \rightarrow c\right)^{\prime}\right] \rightarrow a \text { by (16) }
\end{aligned}
$$


(58)

$$
\begin{aligned}
& b \rightarrow(c \rightarrow a)=[\{0 \rightarrow(c \rightarrow a)\} \rightarrow b] \rightarrow(c \rightarrow a) \quad \text { by (20) } \\
& =\left[\left[(c \rightarrow a)^{\prime} \rightarrow[0 \rightarrow(c \rightarrow a)]\right] \rightarrow[b \rightarrow(c \rightarrow a)]^{\prime}\right]^{\prime} \quad \text { by (I) } \\
& =\left[\left[(c \rightarrow a)^{\prime} \rightarrow\left[(c \rightarrow a)^{\prime} \rightarrow 0^{\prime}\right]\right] \rightarrow[b \rightarrow(c \rightarrow a)]^{\prime}\right]^{\prime} \quad \text { by Lemma } 3.1 \text { (b) } \\
& =\left[\left[(c \rightarrow a)^{\prime} \rightarrow 0^{\prime}\right] \rightarrow[b \rightarrow(c \rightarrow a)]^{\prime}\right]^{\prime} \quad \text { by (31) } \\
& =\left[[0 \rightarrow(c \rightarrow a)] \rightarrow[b \rightarrow(c \rightarrow a)]^{\prime}\right]^{\prime} \\
& =\left[[c \rightarrow(0 \rightarrow a)] \rightarrow[b \rightarrow(c \rightarrow a)]^{\prime}\right]^{\prime} \\
& =\left[\left[c^{\prime \prime} \rightarrow(0 \rightarrow a)\right]^{\prime \prime} \rightarrow[b \rightarrow(c \rightarrow a)]^{\prime}\right]^{\prime} \\
& =\left[\left[\left(0 \rightarrow c^{\prime}\right) \rightarrow(0 \rightarrow a)^{\prime}\right]^{\prime} \rightarrow[b \rightarrow(c \rightarrow a)]^{\prime}\right]^{\prime} \quad \text { by (10) } \\
& =\left[\left[\left(0 \rightarrow\left(0 \rightarrow c^{\prime}\right)\right) \rightarrow(0 \rightarrow a)^{\prime}\right]^{\prime} \rightarrow[b \rightarrow(c \rightarrow a)]^{\prime}\right]^{\prime} \quad \text { by (31) } \\
& =\left[\left[\left((0 \rightarrow a)^{\prime} \rightarrow\left(0 \rightarrow c^{\prime}\right)\right) \rightarrow(0 \rightarrow a)^{\prime}\right]^{\prime} \rightarrow[b \rightarrow(c \rightarrow a)]^{\prime}\right]^{\prime} \quad \text { by (6) } \\
& =\left[\left[\left((0 \rightarrow a)^{\prime} \rightarrow\left(0 \rightarrow c^{\prime}\right)\right) \rightarrow(0 \rightarrow(0 \rightarrow a))^{\prime}\right]^{\prime} \rightarrow[b \rightarrow(c \rightarrow a)]^{\prime}\right]^{\prime} \text { by (31) } \\
& =\left[\left[\left(0 \rightarrow c^{\prime}\right)^{\prime} \rightarrow(0 \rightarrow a)\right] \rightarrow[b \rightarrow(c \rightarrow a)]^{\prime}\right]^{\prime} \\
& =\left[\left[\left(0 \rightarrow c^{\prime}\right)^{\prime} \rightarrow\left(0 \rightarrow a^{\prime \prime}\right)\right] \rightarrow[b \rightarrow(c \rightarrow a)]^{\prime}\right]^{\prime} \\
& =\left[\left[0 \rightarrow\left[\left(0 \rightarrow c^{\prime}\right)^{\prime \prime} \rightarrow a^{\prime}\right]^{\prime}\right] \rightarrow[b \rightarrow(c \rightarrow a)]^{\prime}\right]^{\prime} \\
& =\left[\left[0 \rightarrow\left[\left(0 \rightarrow c^{\prime}\right) \rightarrow a^{\prime}\right]^{\prime}\right] \rightarrow[b \rightarrow(c \rightarrow a)]^{\prime}\right]^{\prime} \\
& =\left[\left[0 \rightarrow\left[\left(c \rightarrow 0^{\prime}\right) \rightarrow a^{\prime}\right]^{\prime}\right] \rightarrow[b \rightarrow(c \rightarrow a)]^{\prime}\right]^{\prime} \\
& =\left[\left[0 \rightarrow\left[\left(c \rightarrow a^{\prime \prime}\right) \rightarrow a^{\prime}\right]^{\prime}\right] \rightarrow[b \rightarrow(c \rightarrow a)]^{\prime}\right]^{\prime} \\
& =\left[\left[0 \rightarrow\left[(c \rightarrow a) \rightarrow a^{\prime}\right]^{\prime}\right] \rightarrow[b \rightarrow(c \rightarrow a)]^{\prime}\right]^{\prime} \\
& =\left[\left[(c \rightarrow a)^{\prime} \rightarrow(0 \rightarrow a)\right] \rightarrow[b \rightarrow(c \rightarrow a)]^{\prime}\right]^{\prime} \\
& =[(0 \rightarrow a) \rightarrow b] \rightarrow(c \rightarrow a)
\end{aligned}
$$

(59)

$$
\begin{aligned}
& a \rightarrow((b \rightarrow a) \rightarrow b)=a \rightarrow[(0 \rightarrow a) \rightarrow b] \quad \text { by (6) } \\
& =[(0 \rightarrow b) \rightarrow a] \rightarrow[(0 \rightarrow a) \rightarrow b] \text { by (58) } \\
& =[(a \rightarrow b) \rightarrow a] \rightarrow[(b \rightarrow a) \rightarrow b] \text { by (6) } \\
& =a \rightarrow b \quad \text { by (9) }
\end{aligned}
$$

(60)

$$
\begin{aligned}
{[(a \rightarrow b) \rightarrow(b \rightarrow c)]^{\prime} } & =\left[\left[(b \rightarrow c)^{\prime} \rightarrow a\right] \rightarrow[b \rightarrow(b \rightarrow c)]^{\prime}\right]^{\prime \prime} & & \text { by (I) } \\
& =\left[(b \rightarrow c)^{\prime} \rightarrow a\right] \rightarrow[b \rightarrow(b \rightarrow c)]^{\prime} & & \\
& =\left[(b \rightarrow c)^{\prime} \rightarrow a\right] \rightarrow(b \rightarrow c)^{\prime} & & \text { by (31) } \\
& =[0 \rightarrow a] \rightarrow(b \rightarrow c)^{\prime} & & \text { by (6) }
\end{aligned}
$$

(61)

$$
\begin{aligned}
(a \rightarrow b) \rightarrow(b \rightarrow a) & =\left[\left[(b \rightarrow a)^{\prime} \rightarrow a\right] \rightarrow[b \rightarrow(b \rightarrow a)]^{\prime}\right]^{\prime} & & \text { by }(\mathrm{I}) \\
& =\left[\left[(b \rightarrow a)^{\prime} \rightarrow a\right] \rightarrow[b \rightarrow a]^{\prime}\right]^{\prime} & & \text { by (131) } \\
& =\left[[0 \rightarrow a] \rightarrow[b \rightarrow a]^{\prime}\right]^{\prime} & & \text { by (6) } \\
& =(b \rightarrow a)^{\prime \prime} & & \text { by (4) } \\
& =b \rightarrow a & &
\end{aligned}
$$


(62)

$$
\begin{aligned}
& {\left[[(a \rightarrow b) \rightarrow c] \rightarrow\left(c^{\prime} \rightarrow a\right)^{\prime}\right] \rightarrow[(a \rightarrow b) \rightarrow c]=\left[0 \rightarrow\left(c^{\prime} \rightarrow a\right)^{\prime}\right] \rightarrow[(a \rightarrow b) \rightarrow c] \quad \text { by ([6) }} \\
& =\left[\left(c^{\prime} \rightarrow a\right) \rightarrow 0^{\prime}\right] \rightarrow[(a \rightarrow b) \rightarrow c] \\
& \text { by Lemma } 3.1 \text { (b) } \\
& =\left[\left(c^{\prime} \rightarrow a\right) \rightarrow[(a \rightarrow b) \rightarrow c]^{\prime}\right] \rightarrow[(a \rightarrow b) \rightarrow c] \text { by (11) } \\
& =\left[\left(c^{\prime} \rightarrow a\right) \rightarrow[(a \rightarrow b) \rightarrow c]^{\prime}\right]^{\prime \prime} \rightarrow[(a \rightarrow b) \rightarrow c] \\
& =[(a \rightarrow(a \rightarrow b)) \rightarrow c]^{\prime} \rightarrow[(a \rightarrow b) \rightarrow c] \quad \text { by }(\mathrm{I}) \\
& =[(a \rightarrow b) \rightarrow c]^{\prime} \rightarrow[(a \rightarrow b) \rightarrow c] \quad \text { by (31) } \\
& =(a \rightarrow b) \rightarrow c \\
& \text { by Lemma } 3.2 \text { (d) }
\end{aligned}
$$

(63)

$$
\begin{aligned}
& {\left[[(a \rightarrow b) \rightarrow c] \rightarrow\left[c^{\prime} \rightarrow(b \rightarrow a)\right]^{\prime}\right] \rightarrow[(a \rightarrow b) \rightarrow c]} \\
& =\left[[((b \rightarrow a) \rightarrow(a \rightarrow b)) \rightarrow c] \rightarrow\left[c^{\prime} \rightarrow(b \rightarrow a)\right]^{\prime}\right] \rightarrow[((b \rightarrow a) \rightarrow(a \rightarrow b)) \rightarrow c] \quad \text { by (61) } \\
& =((b \rightarrow a) \rightarrow(a \rightarrow b)) \rightarrow c \quad \text { by (62) } \\
& =(a \rightarrow b) \rightarrow c \text { by (61). }
\end{aligned}
$$

\section{References}

[1] R. Balbes and PH. Dwinger, Distributive lattices, Univ. of Missouri Press, Columbia, 1974.

[2] S. Burris and H. P. Sankappanavar, A course in universal algebra, Springer-Verlag, New York, 1981. The free, corrected version (2012) is available online as a PDF file at math. uwaterloo.ca/ snburris.

[3] J. M. Cornejo and H. P. Sankappanavar, Implication Zroupoids I. Submitted for publication (2015).

[4] J. M. Cornejo and H. P. Sankappanavar, Implication Zroupoids II. In Preparation.

[5] J. M. Cornejo and H. P. Sankappanavar, Order in Implication Zroupoids. In Preparation.

[6] W. McCune, Prover9 and Mace4, http://www.cs.unm.edu/mccune/prover9/.

[7] H. Rasiowa, An algebraic approach to non-classical logics, North-Holland Publ.Comp., Amsterdam, (1974).

[8] H. P. Sankappanavar, De Morgan algebras: New perspectives and applications, Scientiae Mathematicae Japonicae 75(1): 21-50, 2012. 